\title{
Validation of the BEAMS3D neutral beam deposition model on Wendelstein 7-X
}

To cite this article before publication: Samuel A Lazerson et al 2020 Nucl. Fusion in press https://doi.org/10.1088/1741-4326/ab8e61

\section{Manuscript version: Accepted Manuscript}

Accepted Manuscript is "the version of the article accepted for publication including all changes made as a result of the peer review process, and which may also include the addition to the article by IOP Publishing of a header, an article ID, a cover sheet and/or an 'Accepted Manuscript' watermark, but excluding any other editing, typesetting or other changes made by IOP Publishing and/or its licensors"

This Accepted Manuscript is $\odot 2020$ The Author(s). Published by IOP Publishing Ltd..

During the embargo period (the 12 month period from the publication of the Version of Record of this article), the Accepted Manuscript is fully protected by copyright and cannot be reused or reposted elsewhere.

As the Version of Record of this article is going to be / has been published on a subscription basis, this Accepted Manuscript is available for reuse under a CC BY-NC-ND 3.0 licence after the 12 month embargo period.

After the embargo period, everyone is permitted to use copy and redistribute this article for non-commercial purposes only, provided that they adhere to all the terms of the licence https://creativecommons.org/licences/by-nc-nd/3.0

Although reasonable endeavours have been taken to obtain all necessary permissions from third parties to include their copyrighted content within this article, their full citation and copyright line may not be present in this Accepted Manuscript version. Before using any content from this article, please refer to the Version of Record on IOPscience once published for full citation and copyright details, as permissions will likely be required. All third party content is fully copyright protected, unless specifically stated otherwise in the figure caption in the Version of Record.

View the article online for updates and enhancements. 


\section{Validation of the BEAMS3D neutral beam deposition model on Wendelstein 7-X}

Samuel A. Lazerson ${ }^{1}$, Oliver P. Ford ${ }^{1}$, Carolin Nuehrenberg ${ }^{1}$, Simppa Äkäslompolo ${ }^{1,2}$, Peter Zs. Poloskei', Mike Machielsen ${ }^{3}$ Paul McNeely ${ }^{1}$, Lilla Vanó ${ }^{1}$, Norbert Rust ${ }^{1}$, Sergey Bozhenkov $^{1}$, Tristan W.C. Neelis ${ }^{4}$, Jonathan P. Graves ${ }^{3}$, David Pfefferlé $^{5}$ Annabelle Spanier ${ }^{1}$, Dirk Hartmann ${ }^{1}$, Nikolai Marushchenko $^{1}$, Yuriy Turkin ${ }^{1}$, Matthias Hirsch ${ }^{1}$, Neha Chaudhary $^{1}$, Udo Hoefel ${ }^{1}$, Torsten Stange ${ }^{1}$, Gavin Weir ${ }^{1}$, Novimir Pablant ${ }^{6}$, Andreas Langenberg ${ }^{1}$, Peter Traverso ${ }^{7}$, Pranay Valson ${ }^{1}$, Jens Knauer ${ }^{1}$, Kai Jakob Brunner ${ }^{1}$, Ekkehard Pasch $^{1}$, Marc Beurskens ${ }^{1}$, Hannes Damm ${ }^{1}$, Golo Fuchert ${ }^{1}$, Philipp Nelde ${ }^{1}$, Evan Scott ${ }^{1,8}$, Uwe Hergenhahn ${ }^{1}$, Andrea Pavone $^{1}$, Kian Rahbarnia ${ }^{1}$, Tamara Andreeva ${ }^{1}$, Jonathan Schilling $^{1}$, Christian Brandt ${ }^{1}$, Ulrich Neuner ${ }^{1}$, Henning Thomsen $^{1}$, Marcin Jakubowski ${ }^{1}$, Adnan $\mathrm{Ali}^{1}$, Yu Gao ${ }^{1}$, Holger Niemann ${ }^{1}$, Aleix Puig Sitjes ${ }^{1}$, Ralf Koenig ${ }^{1}$, Robert C. Wolf ${ }^{1}$ and the W7-X Team

${ }^{1}$ Max-Planck-Institut für Plasmaphysik, 17491 Greifswald, Germany

${ }^{2}$ Aalto University, 02150 Espoo, Finland

${ }^{3}$ École Polytechnique Fédérale de Lausanne, Swiss Plasma Center, CH-1015

Lausanne, Switzerland

${ }^{4}$ Eindhoven University of Technology, 5600 Eindhoven, Netherlands

${ }^{5}$ University of Western Australia, 6009 Crawley, Australia

${ }^{6}$ Princeton Plasma Physics Laboratory, 08543 Princeton, USA

${ }^{7}$ Auburn University, 36849 Auburn, USA

${ }^{8}$ University of Wisconsin, 53715 Madison, USA

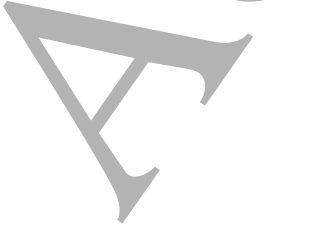

E-mail: samuel.lazerson@ipp.mpg.de

Abstract. The neutral beam deposition model in the BEAMS3D code is validated against neutral beam attenuation data from Wendelstein 7-X (W7-X). A set of experimental discharges where the neutral beam injection system of W7$\mathrm{X}$ was utilized were reconstructed. These discharges scanned the magnetic configurations and plasma densities of W7-X. The equilibrium reconstructions were performed using STELLOPT which calculates three-dimensional self-consistent ideal magnetohydrodynamic equilibria and kinetic profiles. These reconstructions leveraged new capabilities to incorporate electron cyclotron emission and X-ray imaging diagnostics in the STELLOPT code. The reconstructed equilibria and profiles served as inputs for BEAMS3D calculations of neutral beam deposition in W7-X. It is found that if reconstructed kinetic profiles are utilized, good agreement between measured and 
W'-X BEAMS3D Validation

simulated beam attenuation is found. As deposition models provide initial conditions for fast-ion slowing down calculations, this work provides a first step towards validating our ability to predict fast ion confinement in stellarators.

Submitted to: Nuclear Fusion

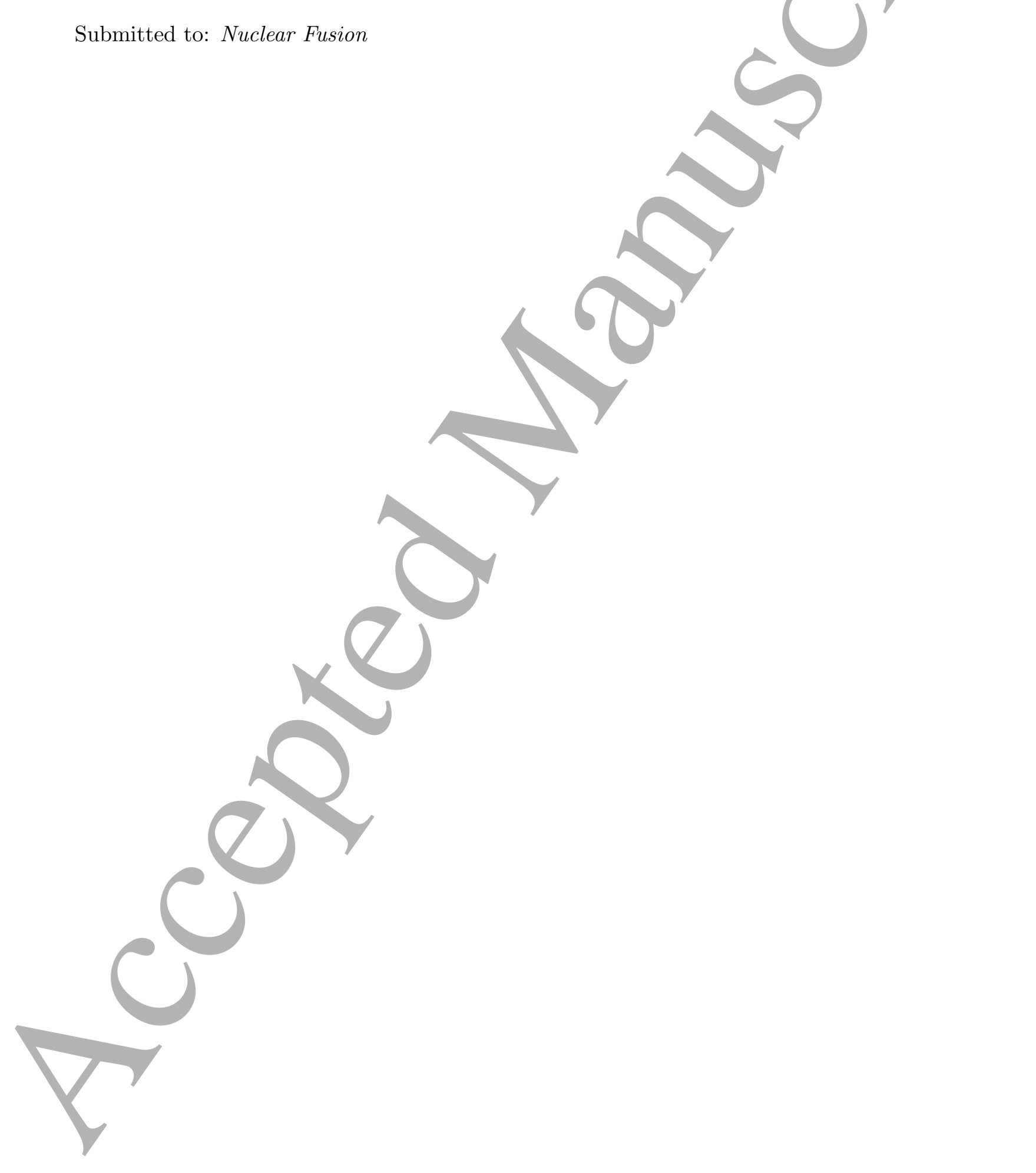




\section{W7-X BEAMS3D Validation}

\section{Introduction}

Validation of numerical models against experimental data requires that the inputs to said models accurately reflect the state of the experiment to which comparisons are being made. In the case of neutral beam modeling, the plasma profiles (electron density, electron temperature, ion temperature, effective ion charge number, and radial electric field), beam geometry, and magnetic fields serve as the model inputs. In this work, the plasma profiles and magnetic fields are matched to experimental measures through three dimensional equilibrium reconstructions of Wendelstein 7-X (W7-X) plasmas [1] using the STELLOPT code [2]. The resulting profiles and self-consistent three dimensional ideal magnetohydrodynamic equilibria serve as inputs to the BEAMS3D neutral beam code [3]. Simulations with this code are compared against measurements of neutral beam attenuation allowing validation of the neutral beam model. Validated models of neutral beam deposition are key to validating energetic particle slowing down models. These are in-turn key to validating wall losses and thereby providing validated tools for energetic particle confinement in stellarators. With a validated tool for energetic particle confinement one can make predictive estimates of alpha confinement in a stellarator reactor.

Commissioning of the first neutral beam line on W7-X during the first divertor campaign allowed preliminary assessment of neutral beam injection (NBI) [4]. Two of four sources were installed in the N121 beam box allowing for counter-injection of $55 \mathrm{keV} \mathrm{H}^{0}$ particles (here counter implies a direction in which current drive raises the rotational transform). In this work we concern ourselves with six discharges which scan both magnetic configuration and plasma density. In five of these discharges, the NBI was fired into plasmas supported by approximately $4 M W$ of X-mode electron cyclotron resonance heating (ECRH). A sixth discharge is also considered which is performed without any additional ECRH and demonstrates NBI operation over a wide range of densities. Measurements of beam attenuation provide a basis for validating the neutral beam deposition model in the BEAMS3D code.

In order to provide the BEAMS3D code with an accurate depiction of the plasma, three dimensional equilibrium reconstructions of the W7-X plasma discharges were performed. The process of equilibrium reconstruction is one in which the inputs of an equilibrium model are varied such that a match between synthetic diagnostic measures and measured quantities is found. In this work, we utilize the STELLOPT code which has previously been applied as a reconstruction tool for the plasmas of Wendelstein 7-AS [5], the Large Helical Device [6], and DIII-D [7]. Additionally, it has been used to assess the effect of three-dimensional coils in ITER [8]. The equilibrium model in STELLOPT is the variational moments equilibrium code (VMEC) which has been widely used in the stellarator community [9]. This code enforces the constraint of continuously nested magnetic flux surfaces through, albeit weak, shielding currents [10]. The DIAGNO code [11] provides a synthetic diagnostic model for flux loops and Rogowski coils on W7-X. A newly developed interface between STELLOPT and the TRAVIS code provides a 


\section{W7-X BEAMS3D Validation}

synthetic electron cyclotron emission (ECE) model [12]. The X-Ray Imaging Crystal Spectrometer (XICS) and line-integrated effective ion charge $\left(Z_{\text {eff }}\right)$ diagnostics have been included in the STELLOPT reconstruction model.

With the reconstructed profile data and self-consistent VMEC equilibria the deposition profile of the BEAMS3D code can be compared against experimental measurements of beam attenuation. Spectroscopic diagnostics in W7-X view the beamline and provide measurements of the attenuation of the neutral beam [13]. In/addition, partial measurements with infrared cameras help to verify the beam geometry. In the next section we describe the experimental parameters and codes used in this work in more detail. In sections 3 and 4 the equilibrium reconstructions and beam attenuation calculations are discussed. Finally in section 5 we discuss the results and look at future work and applications.

\section{Methods}

A set of discharges varying magnetic configuration and electron density are considered. These are all nominally $4 M W$ ECRH discharges with additional NBI heating, with the exception of one in which ECRH is only used for plasma startup. Preliminary analysis is performed using a rapid profile fitting algorithm which does not compute self-consistent three-dimensional equilibria. Thís accelerates full three-dimensional equilibrium reconstruction by providing a good first approximation. Fully self-consistent three dimensional magnetohydrodynamical equilibria are then calculated using the STELLOPT code. In doing so diagnostics such as electron cyclotron emission (ECE) and magnetic diagnostics may be included in the reconstruction. This step provides input parameters for the BEAMS3D neutral beam injection and energetic particle following code. These simulations provide data allowing comparisons to be made with measured beam parameters.

\subsection{Experimental overview}

Table 1. Overview of discharges considered in this work. Discharges were chosen to provide a scan of plasma density, magnetic configurations, and heating schemes.

\begin{tabular}{c|c|c|c|c|c|} 
Shot ID & Mag. Config. & Heating & Central Density $\left[\mathrm{m}^{-3}\right]$ & Edge Iota & Mirror Ratio \\
\hline 20180812.12 & Standard & ECRH + NBI & $5 \times 10^{19}$ & $5 / 5$ & 0.04 \\
20180812.17 & Standard & ECRH + NBI & $2 \times 10^{19}$ & $5 / 5$ & 0.04 \\
20180812.19 & Standard & ECRH + NBI & $8 \times 10^{19}$ & $5 / 5$ & 0.04 \\
20180822.12 & High Iota & ECRH + NBI & $5 \times 10^{19}$ & $5 / 4$ & 0.05 \\
20180823.12 & High Mirror & ECRH + NBI & $5 \times 10^{19}$ & $5 / 5$ & 0.10 \\
20181009.43 & High Mirror & NBI & $20 \times 10^{19}$ & $5 / 5$ & 0.10
\end{tabular}



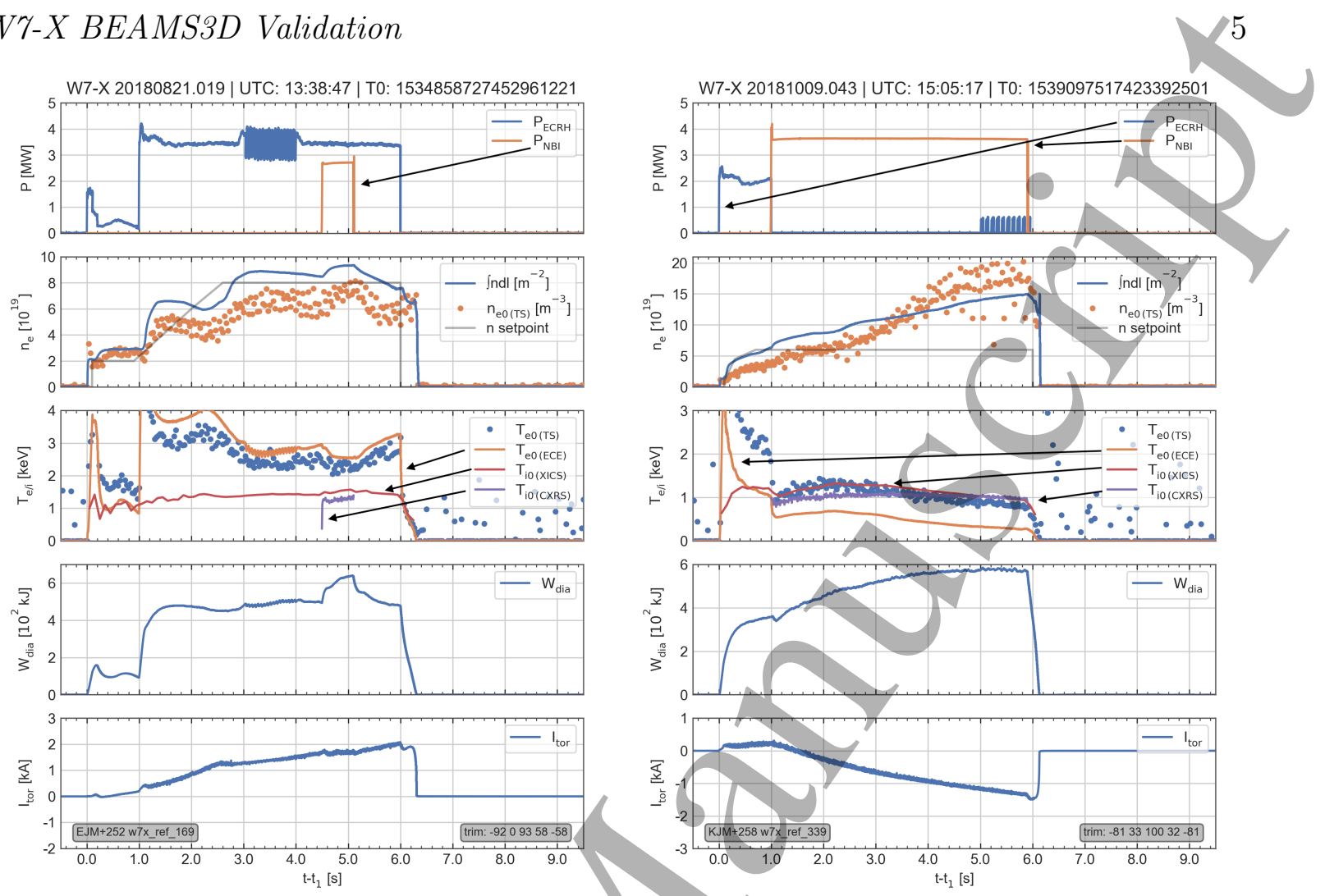

Figure 1. Overview plots depicting an ECRH+NBI discharge (left) and one purely supported by NBI (right). In the ECRH, discharge the NBI is fired around $4.5 \mathrm{~s}$ into the discharge.

For the purposes of this work six discharges (Table 1) were considered. In the majority of these discharges the NBI was added on top of $4 M W$ of ECRH. In discharge 20181009.43, $2 M W$ of ECRH was used to initiate the plasma for $1 s$ at which point it was turned off. Simultaneous with the ECRH turn-off, the NBI system fired for $5 s$, creating a discharge purely supported by NBI. The standard and high mirror configurations utilize the $m / n=5 / 5$ island chain while the high iota configuration has a $m / n=5 / 4$ edge island chain. The high iota and standard magnetic configurations have mirror ratios of $\sim 0.05$ while the high mirror configuration has a mirror ratio of $\sim 0.10$. The mirror ratio is defined as the ratio of the magnetic field strength across a half-field period (from high to low field).

Figure 1 depicts an overview of these two types of discharge. The inclusion of NBI into an ECRH discharge results in a slight increase in the electron density with an abrupt change in the measured diamagnetic energy. Little to no change in ion temperature occurs during these discharges, which is consistent with attempts to increase the ion temperature through additional ECRH and increased densities. The discharge supported by pure NBI heating shows a continual density rise, with peaking of the density profile evident after $4 s$ into the discharge. The peaking can be inferred from the core Thomson scattering value rising above the line integrated density. The ion and electron temperatures show a slight decrease over the duration of the discharge 
W7-X BEAMS3D Validation $\quad<6$

with mean values around $1 \mathrm{keV}$.

\subsection{Rapid profile fitting}

In order to aide in analysis a rapid profile analysis tool for W7-X was developed, WAPID_FIT. This code use pre-computed diagnostic lookup-tables to fit simple profile functions to various diagnostic measures. These lookup tables are based on diagnostic geometry and VMEC equilibria fitted to the W7-X vacuum last closed flux surfaces. While this introduces a number of approximations, it significantly increases the computational speed of profile fitting. Within a couple of seconds electron temperature, electron density and ion temperature profiles can be generated from experimental measures. This speedup allows entire discharges to be analyzed.

The diagnostic lookup tables are generated from zero current, zero pressure VMEC equilibria, and contain inversion data for a multitude of diagnostics. The equilibria themselves are produced by fitting boundary shapes to the vacuum last closed flux surface for a given magnetic configuration. Care is taken to ensure that the fixed boundary VMEC calculation captures the magnetic axis location, and that the PHIEDGE parameter is chosen to match the vacuum field level used in experiments (usually $2.52 \mathrm{~T}$ on axis). Figure 2 depicts such a fit. For diagnostics, like charge exchange and Thomson scattering, the coordinates of measurement points in Cartesian space are mapped into the equilibrium space $\left(s=\Phi / \Phi_{\text {edge}}\right.$, where $\Phi$ is toroidal flux) and recorded in the lookup file for a given configuration. For line-integrated quantities such as interferometry and the XICS system, 256 data points across each channel of the diagnostic are inverted and recorded. The local poloidal angle of data points are stored 


\section{W7-X BEAMS3D Validation}

so that poloidal variation may be included in the future.

Fits are produced for time slices centered around the firing of the Thomson scattering laser system. The electron density is first fit in a normalized sense using a truncated Gaussian formula of the form

$$
f_{\text {gauss trunc }}(\rho)=C_{0}+\frac{C_{1}}{1-e^{-\left(1 / C_{2}\right)^{2}}}\left(e^{-\left(\rho / C_{2}\right)^{2}}-e^{-\left(1 / C_{2}\right)^{2}}\right),
$$

where $C_{k}$ are coefficients and $\rho=\sqrt{s}$ is the radial coordinate. A scaling coefficient is then fit using this profile and the line integrate density as obtained from the interferometer signal.The electron temperature as measured by Thomson scattering is then fit using a two-power function of the form

$$
f_{\text {two power }}(\rho)=C_{0}\left(1-\rho^{C_{1}}\right)^{C_{2}} \text {. }
$$

An 8 knot spline is used to represent an effective emissivity $(\epsilon)$ and fit to the measured brightness for each of the 26 XICS channels [14]. The XICS measured ion temperature is a convolution of the effective ion temperature and this emissivity. The signals are modeled with

$$
\begin{aligned}
& f_{\text {Brightness channel }}^{X I C S}=\int \epsilon(\rho) d l \\
& f_{T_{i} \text { channel }}^{X I C S}=\int \epsilon(\rho) T_{i}(\rho) d l \\
& f_{T_{e} \text { channel }}^{X I C S}=\int \epsilon_{\text {sat }} d l=\int \epsilon(\rho) F_{f i t}\left(T_{e}\right) d l,
\end{aligned}
$$

where the integration is over the channel line of sight, $T_{i}$ is the ion temperature, $T_{e}$ is the electron temperature, and $\epsilon$ (the/local emissivity) is held fixed during the fit of $T_{i}$ and $T_{e}$. The quantity $F_{f i t}$ is a function of electron temperature determined from experimental spectroscopic measurements. A two-power profile is used for the ion and electron temperature profiles.

During NBI operation the charge exchange recombination system (CXRS) measures parameters along the beam-line, including the ion temperature [13]. A two-power profile is used for the fit of this data, with the lookup table providing the mapping between cartesian space and $\rho$ for each channel.

The WAPID_FIT code provides a quick and simple automated fit of a subset of the W7-X diagnostic data. An example of the fit parameters for a single time slice is shown in figure 3 . The upper left plot shows the various ion and electron temperature profiles plotted against the electron temperatures as measured by the Thomson scattering system. The electron temperature as fit from Thomson and XICS appear in good agreement with the XICS being slightly higher in temperature than the Thomson. The ion temperatures between CXRS and XICS show a large disagreement in the core. In the lower center plot, the ion temperature calculated by XICS has been projected onto the coordinates of the CXRS system. This indicates that the disagreement is not a simple matter of diagnostic interpretation. The profile data from WAPID_FIT is used as an initial condition for full equilibrium reconstructions using the STELLOPT code. 
W'-X BEAMS3D Validation 18
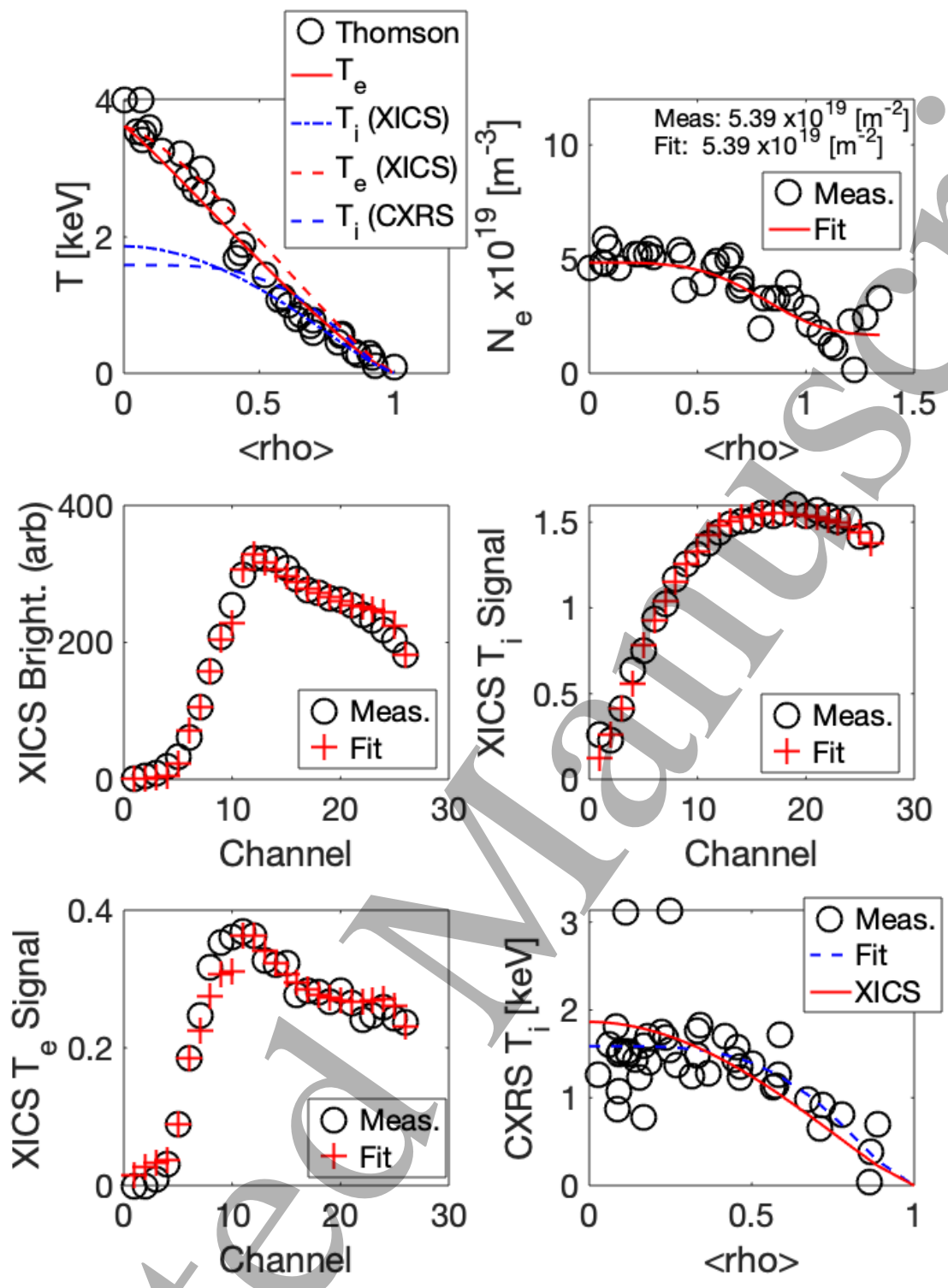

Figure 3. Overview of a WAPID_FIT analysis for a single time slice. Each frame corresponds to a different diagnostic. Simulated values show as red crosses $(+)$ with measured values/as circles (o). The XICS channels go from edge (1) to core views (26). Where appropriate the radial profiles have been shown.

\subsection{Equilibrium reconstruction with STELLOPT}

A full three-dimensional equilibrium reconstruction can be provided by the stellarator optimization (STELLOPT) code. The STELLOPT code performs a chi-squared $\left(\chi^{2}\right)$ minimization of a set of user selectable target functionals, where the optimized quantities are the inputs to the VMEC equilibrium code. The chi-squared functional has the form

$$
\chi^{2}\left(x_{1} \ldots x_{j}\right)=\sum_{i=1}^{m}\left[\frac{y_{i}-f_{i}\left(x_{1}, \ldots x_{j}\right)}{\sigma_{i}}\right]^{2}
$$




\section{W7-X BEAMS3D Validation}

where $j$ is an index over the input parameters $x, i$ is summed over the $m$ target functionals, $y_{i}$ are the desired values of the functionals, $f_{i}$ are the functionals, and $\sigma_{i}$ are weights on the functionals. Minimization in this work is performed using a modified Levenberg-Marquardt algorithm. The result is an equilibrium model with self-consistent pressure, current and magnetic field which has been fit to various diagnostic measures.'

Table 2. Number of free parameters in the equilibrium reconstruction.

\begin{tabular}{c|c|c} 
Toroidal Current & Pressure Scaling Factor & Enclosed Toroidal Flux \\
1 & 1 & 1 \\
\hline Current Density Profile & Electron Density Profile & Electron Temperature Profile \\
6 & 5 & 4 \\
\hline Ion Temperature Profile & XICS Emissivity Profile & Effective Ion Charge \\
4 & 8 & 1
\end{tabular}

When performing an equilibrium reconstruction the code varies input parameters to the VMEC code related to the pressure profile, current density profile, and magnetic configuration. The pressure profile is treated as a composite of temperature and density profiles where it is assumed all quantities are functions of the normalized toroidal flux

$$
p(s)=n_{e}(s) k_{B}\left(T_{e}(s)+T_{i}(s) / Z_{e f f}(s)\right) .
$$

In this equation $s=\Phi / \Phi_{\text {edge }}$ is the normalized toroidal flux, $p$ the plasma pressure, $n_{e}$ the electron density, $k_{B}$ Boltzmann's constant, $T_{e}$ the electron temperature, $T_{i}$ the ion temperature, and $Z_{\text {eff }}$ the effective ion charge number. The electron and ion temperature are modeled with 6 knot splines. The last knot at $s=1.0$ is held fixed at zero. The electron density is modeled as a 5 knot spline however, unlike the temperature profiles, the edge value at $s=1.0$ is allowed to vary. The effective ion charge number is modeled as a constant across the plasma. The current density profile is modeled with a power series. As the discharges were low plasma beta, fixed boundary equilibria were used in this work but the enclosed toroidal flux was allowed to vary. Table 2 provides an overview of the varied quantities.

The target functionals/included for reconstruction include magnetic diagnostics (flux loops and Rogowski's) [15, 16], Thomson scattering [17, 18], ECE [19], XICS [14], line integrated Z-effective[20], and CXRS [13]. Figure 5 depicts the diagnostic measurements mapped into a single field period of the device. The magnetic diagnostic response is modeled using the DIAGNO code. Eight saddle coils in one field period along with the uncompensated diamagnetic loops are targeted. A subset of the segmented Rogowski coils is considered in this work along with the full Rogowski coil. Both the high and low field side Thomson channels are considered in this work and coordinate inversion is handled by the STELLOPT code.

Recently the STELLOPT code has been upgraded to include a synthetic diagnostic for the XICS system where equations 3, 4, and 5 are solved. The line integrals of the 
effective emissivity, ion temperature, electron temperature, and rotation are modeled using the same line integration methods employed for the line integrated density as determined by the interferometer. The code approximates the line integral using 256 points across the plasma for each of the channels. In this work we have neglected the measurement of poloidal rotation as it does not affect the VMEC equilibrium or deposition model in BEAM3D [21]. While not needed for the deposition calculation the radial electric field changes particle orbits through the $\vec{E} \times \vec{B}$ drift term.

The inclusion of the ECE diagnostic in STELLOPT has been made possible by a coupling to the TRAVIS code. TRAVIS is a ray tracing code which simulates the radiated power as observed by the ECE system on W7-X. In this work both the Xmode and O-Mode contributions to the radiated power are considered. The use of the TRAVIS model is necessary as it has been found that the cold resonances do not appear to provide a good approximation to W7-X plasmas. 


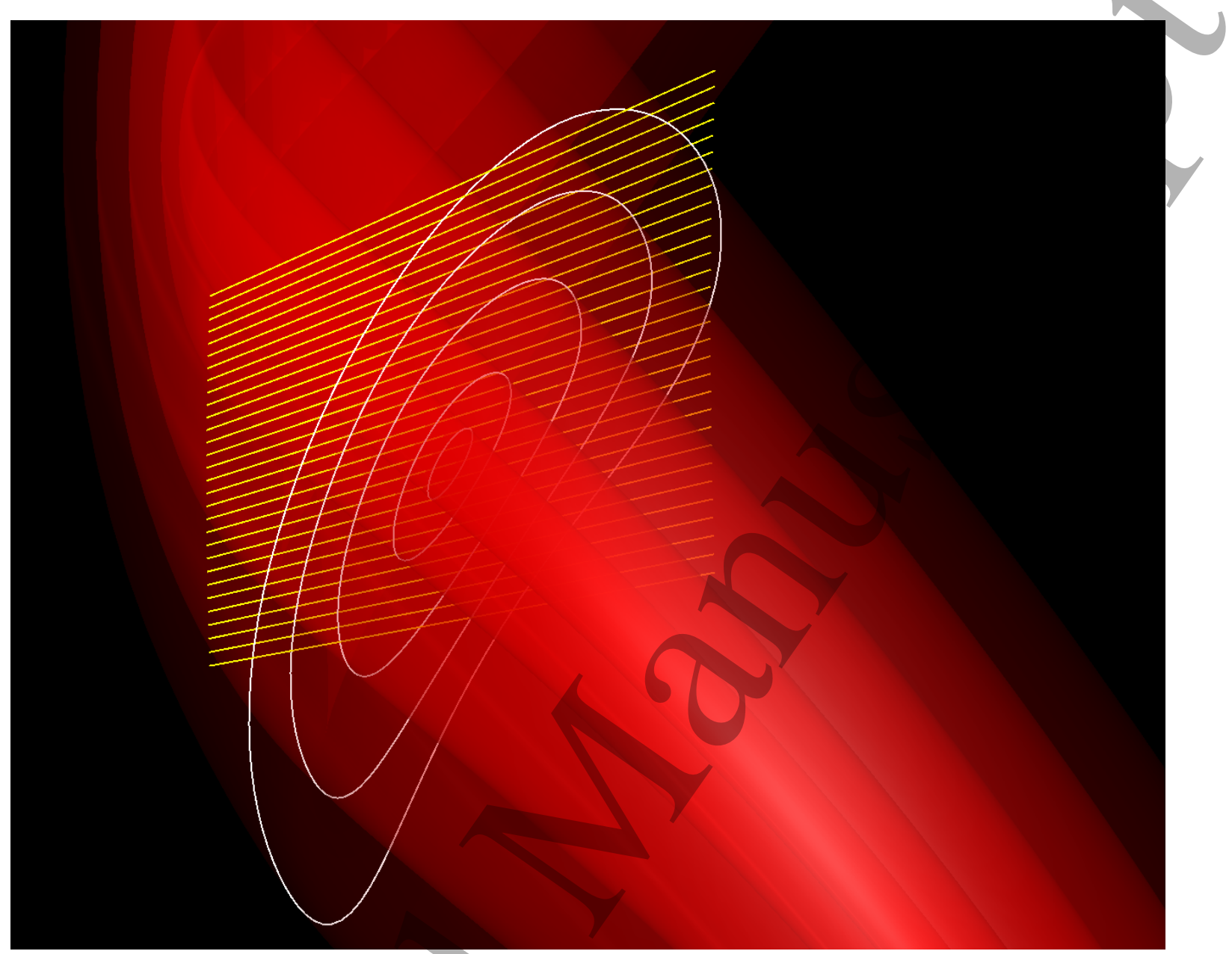

Figure 5. Depiction of the W7-X XICS chord lines (yellow) as they pass through the plasma. A VMEC equilibrium has been utilized to visualize the plasma in red. A select set of surfaces passing through the chord plane have been highlighted in white.

In this work the current density is modeling using profile of the form

$$
j(s)=-4 \sqrt{s}+4 \sqrt{s^{3}}+\sum_{n=0}^{6} a_{n} s^{n} .
$$

In this equation the first two terms are chosen to mimic a bootstrap-like current density profile in $\rho=\sqrt{s}$. This bootstrap-like profile is zero at $s=0$ and $s=1$ with a maximum value at $s=0.25(\rho=0.5)$. The coefficients $\left(a_{n}\right)$ are allowed to vary during the reconstruction to account for driven and induced contributions to to the total current density profile. The profile itself is renormalized to the total toroidal current which is also allowed to vary during reconstruction. The necessity of this second term is due to the long current relaxation time in W7-X and the presence of neutral beam and electron-cyclotron current drive. 


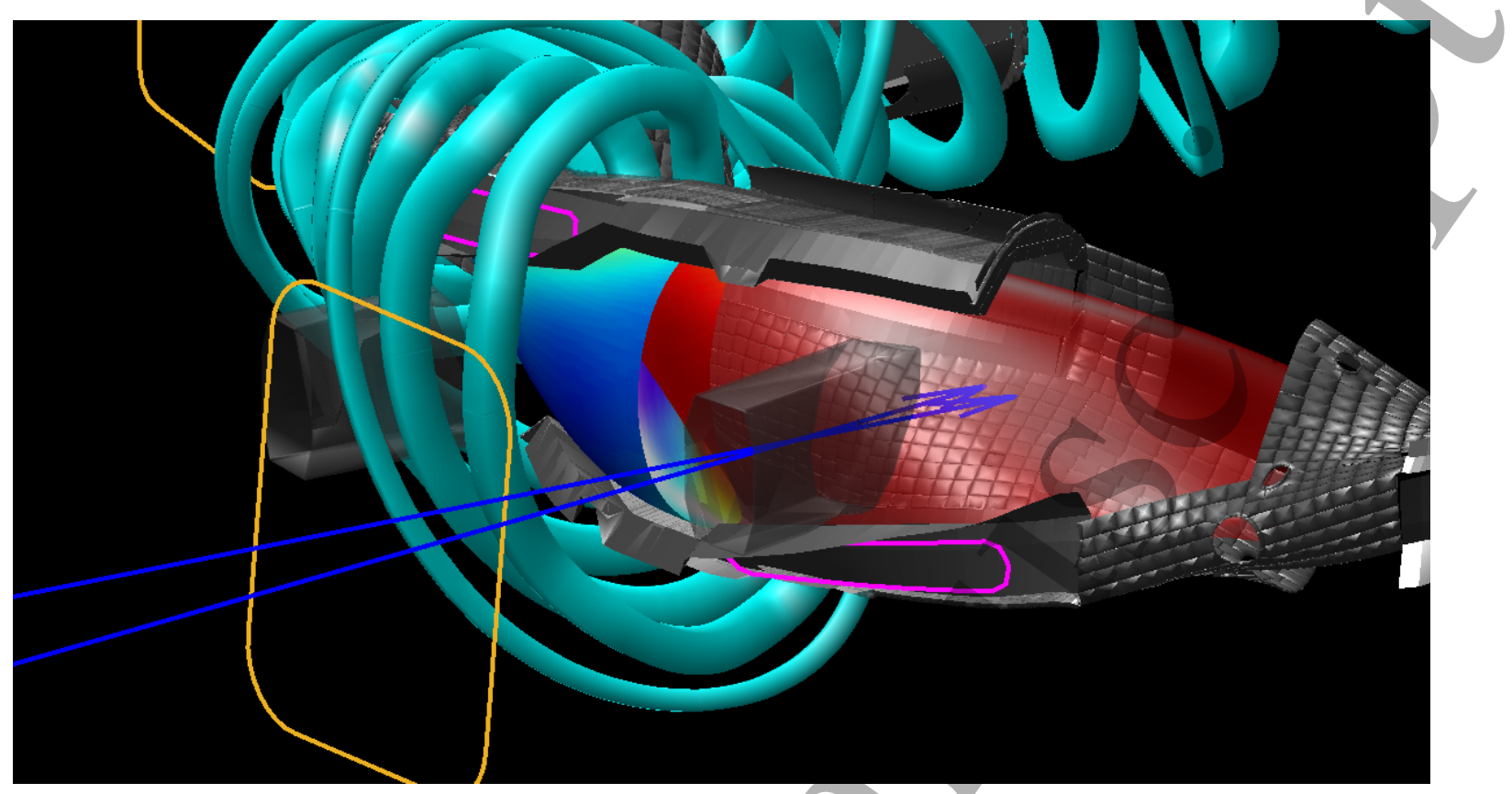

Figure 6. Depiction of the NBI geometry for source 7 and 8 . The blue arrows depict the centerline of the beam lines for the sources. The superconducting coils (cyan) and VMEC last closed flux surface have been depicted for reference. The perturbative auxiliary coils are depicted in yellow and magenta, respectively.

\subsection{Deposition modeling with BEAMS3D}

The BEAMS3D code is a 3D energetic particle code which simulates both the gyrocenter orbits of energetic particles and their/generation by neutral beam injection. The reconstructed VMEC equilibrium and profiles serve as inputs to the BEAMS3D neutral beam code. In this work we focus on the validation of the neutral beam deposition model in the code.

The BEAMS3D code utilizes a cylindrical grid upon which all computations are preformed. In this work a grid of 128 radial, 128 vertical, and 32 toroidal grid points are considered. The radial grid has an extent of $R=[4.5,7] m$, a vertical extent of $Z=[-1.5,1.5] \mathrm{m}$, and extends toroidally over one field period $\phi=[0,2 \pi / 5] \mathrm{rad}$. During initialization each grid point is inverted into the $s, u, v$ space of the VMEC equilibrium (where $u$ is a poloidal-like angle and $v$ is a toroidal-like angle). Points lying inside the VMEC equilibrium have the components of the magnetic field (and normalized toroidal flux) taken directly from VMEC. Points outside the equilibrium are assumed to be in vacuum and have their magnetic field taken from a linear superposition of the fields arising from the vacuum field coils and the plasma current (via virtual casing). The electron density, electron temperature, ion temperature, and effective charge number are also placed on this grid using the $s$ coordinate obtained from VMEC (values outside the VMEC domain are taken to be zero). Finally, a three dimensional cubic Hermite spline is made over these grids. This is done to provide a smooth transition to the vacuum region and avoids ringing of the quantities. 
W7-X BEAMS3D Validation
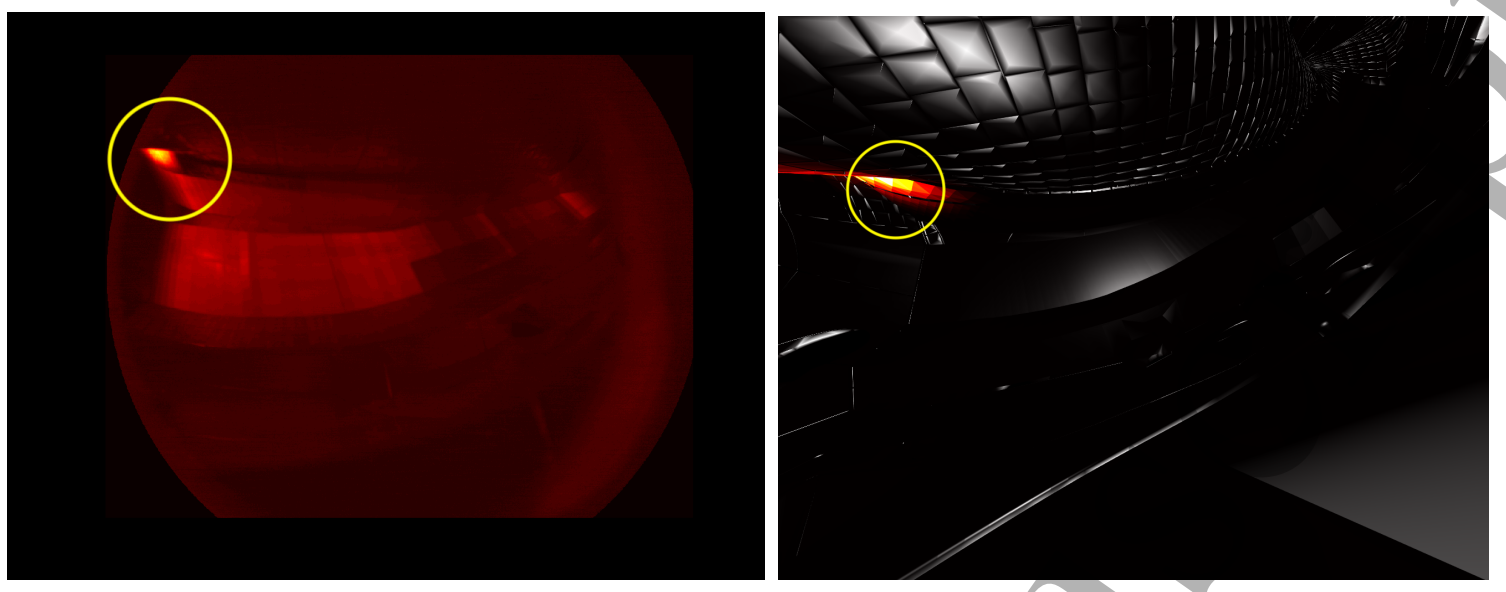

Figure 7. Experimental (left) and simulated (right) divertor IR camera image showing agreement between simulated and measured beam dump hot spot. The hotspot has been highlighted via a circle.

As the code is Monte-Carlo in nature, an ensemble of neutral particles is tracked into the plasma for each source in the neutral beam, and each energy of the beam. The neutral beams in W7-X are composed of two RF sources (S7 and S8) which are accelerated by a $55 \mathrm{kV}$ grid, producing hydrogen populations at $E=55 \mathrm{keV}$, $E / 2=27.5 \mathrm{keV}$, and $E / 3=18.3 \mathrm{keV}$. Thus a total of 6 beam populations are considered in this work. Figure 6 shows the NBI geometry for the two sources relative to the W7-X plasma and wall structure. The sources fire across each other resulting in one beam-line being slightly more radial and another being slightly more tangential. Recently the code has been extended to/include the acceleration grid geometry and the effect of the three dimensional duct geometry. The acceleration grids are composed of 774 beamlets which span a region $\sim 24 \mathrm{~cm}$ across and $\sim 50 \mathrm{~cm}$ in height. The upper and lower half of each grid are slightly angled to focus the beam. Power fractions of $55 \%, 31 \%$, and $14 \%(E, E / 2, E / 3)$ were assumed for these simulations although, this parameters do not influence the deposition results (1.8 $\mathrm{MW}$ assumed source power).

In order to assess the accuracy of the neutral beam geometry a simulation was performed with no plasma and compared to IR camera images of the NBI system firing into the torus with no plasma breakdown. This IR camera view was optimized for divertor safety, as a result only a partial view of one source hot-spot was possible. Figure 7 shows camera data and synthetic views using simulation data. In general, good agreement was seen for the base parameters which is consistent with other works $[22]$.

Neutral beam deposition is calculated by following particle trajectories in three stages. In the first stage a particle is followed into the plasma recording the point along the trajectory just outside the equilibrium domain but within $1 \mathrm{~cm}$ of the equilibrium domain. In the second step a similar point is found where the neutral trajectory leaves the equilibrium domain. In the third step, the electron temperature, ion temperature, electron density and effective ion charge number are evaluated at 256 points between 
W'-X BEAMS3D Validation 14

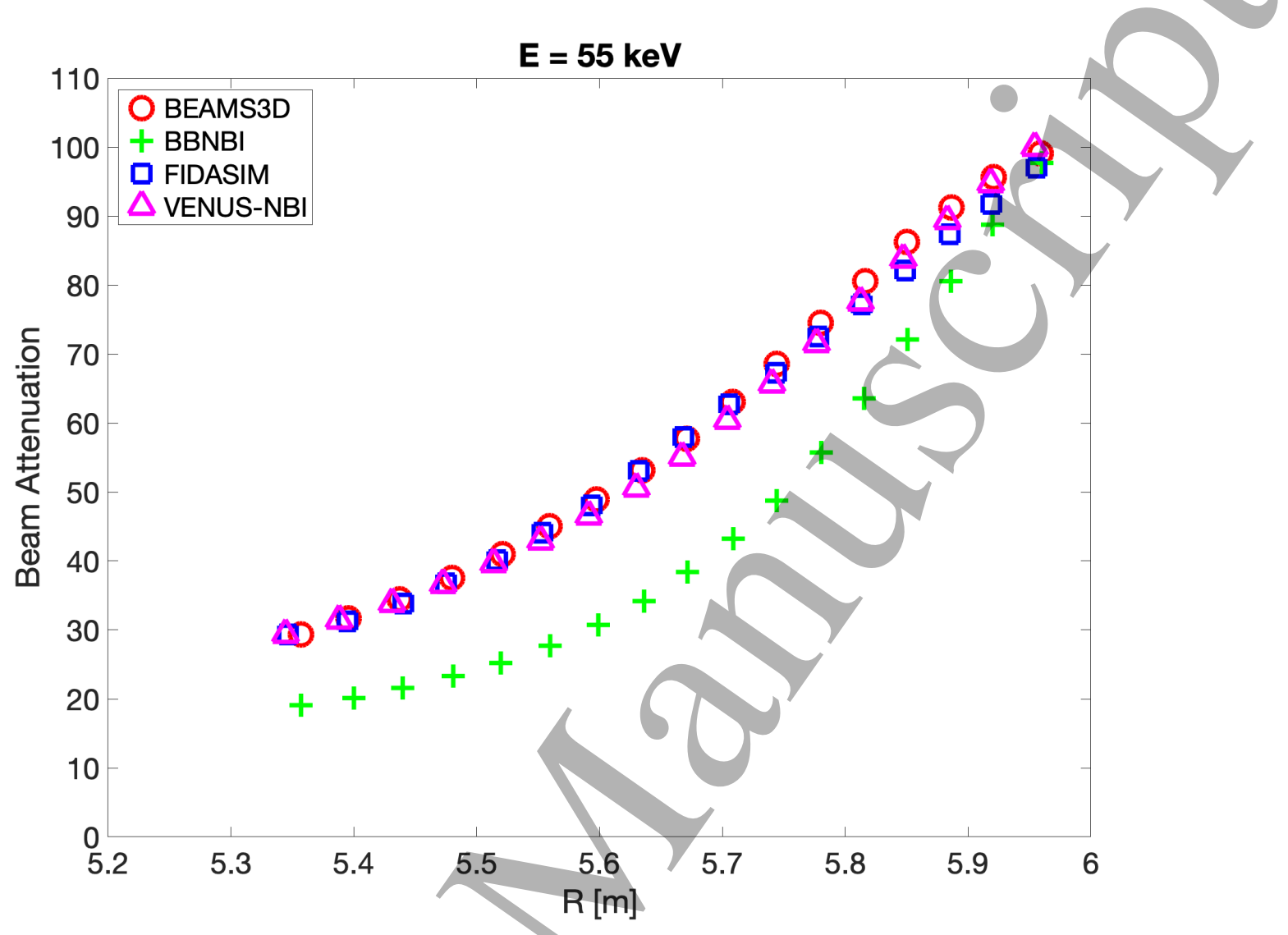

Figure 8. Synthetic diagnostic for neutral beam attenuation for BEAM3D (o), FIDASIM (square), VENUS-NBI (triangle), and BBNBI $(+)$ codes. Discrepancies between the codes are attributed to handling of background grids and ionization algorithms. Attenuation is relative to the first channel at $R \sim 5.95 \mathrm{~m}$.

these two end points. These values are then used to calculate the ion-impact $\left(\left\langle\sigma_{i i} v\right\rangle\right)$, electron-impact $\left(\left\langle\sigma_{e i} v\right\rangle\right)$, and charge exchange cross-sections $\left(\left\langle\sigma_{c x} v\right\rangle\right)$ using the ADAS reaction tables. The probability of ionization is then calculated along the trajectory with the form

$$
f(x)=\int_{0}^{x} f e^{-\Delta t\left(\left\langle\sigma_{i i} v\right\rangle+\left\langle\sigma_{e i} v\right\rangle+\left\langle\sigma_{c x} v\right\rangle\right) n_{e}} d x
$$

where the integral is meant to imply a cumulative sum, $n_{e}$ is the local electron density and $\Delta t$ is the time step. It should be noted that the presence of $n_{e}$ in this equation is due to normalizations coming from the ADAS reaction tables. The ion impact and charge exchange coefficients are functions of the effective charge number. A random number between 0 and 1 is chosen for each particle and when the probability of ionization exceeds this number the particle is considered ionized. Particle which do not exceed this number are then followed to the wall and their collision with the wall is recorded. When a particle is ionized the gyrocenter is determined by assuming a random gyro-phase and stepping by the local plasma parameters accordingly.

In the experiment the beam attenuation is measured using spectroscopic 
measurements of the $n=3$ emission for multiple sight lines crossing the beam geometry. In order to compare simulations with measurements a synthetic diagnostic has been developed. The positions at which the Monte-Carlo neutral particles ionize are binned along sight-lines of the diagnostic to provide a comparable measure. Figure 8 depicts this synthetic beam attenuation measurement for the BEAMS3D, FIDASIM [23, 24], VENUS-NBI [25], and BBNBI codes [26]. FIDASIM and BEAMS3D utilize an interface to ADAS data [27], while BBNBI and VENUS-NBI uses the Suzuki model for charge exchange of the neutral particles [28]. In these plots, attenuation refers to the amount of beam remaining, thus $100 \%$ of the beam is measured at the right hand side of the plot, where injection takes place. In the W7-X experimental profiles (documented in the next section) the plasma density is finite at the equilibrium boundary while electron and ion temperatures are taken to go to zero at the equilibrium boundary. This results in large beam stopping coefficients, requiring careful treatment of these regions to prevent anomalously high values of particle deposition. For BBNBI this results in anomalously low values of shine through, and thus the discrepancy in the plot. Assessment and correction of this effect will be the subject of future work. It should also be noted that relative beam attenuation profiles are plotted for comparison with measurements which have not yet been absolutely calibrated.

\section{Reconstructed Equilibria}

Table 3. Overview of reconstructed central electron temperature $\left(T_{e 0}\right)$, central ion temperature $\left(T_{i 0}\right)$, central electron density $\left(n_{e 0}\right)$, total toroidal current $\left(I_{t o r}\right)$, and average plasma beta $(<\beta\rangle)$ for the time slices considered in this work.

\begin{tabular}{|c|c|c|c|c|c|c|} 
Shot ID & Time Slice [ms] & $T_{e 0}[\mathrm{keV}]$ & $T_{i 0}[\mathrm{keV}]$ & $n_{e 0}\left[\mathrm{~m}^{-3}\right]$ & $I_{\text {tor }}[\mathrm{kA}]$ & $<\beta>[\%]$ \\
\hline 20180812.12 & 5100 & 3.33 & 1.72 & $6.37 \times 10^{19}$ & -2.63 & 0.5 \\
20180812.17 & 4600 & 4.70 & 1.68 & $2.80 \times 10^{19}$ & -1.27 & 0.3 \\
20180812.19 & 4800 & 2.30 & 1.71 & $11.3 \times 10^{19}$ & -1.48 & 0.6 \\
20180822.12 & 6600 & 2.55 & 1.76 & $5.82 \times 10^{19}$ & 1.15 & 0.4 \\
20180823.20 & 5200 & 2.39 & 1.57 & $7.39 \times 10^{19}$ & -0.30 & 0.4 \\
20181009.43 & 1500 & 1.15 & 1.30 & $10.5 \times 10^{19}$ & 0.05 & 0.4 \\
20181009.43 & 2500 & 1.25 & 1.40 & $11.8 \times 10^{19}$ & 0.50 & 0.4 \\
20181009.43 & 3500 & 1.10 & 1.27 & $17.0 \times 10^{19}$ & 0.92 & 0.5 \\
20181009.43 & 4500 & 0.96 & 1.11 & $21.1 \times 10^{19}$ & 1.07 & 0.6 \\
20181009.43 & 5500 & 0.84 & 1.01 & $27.8 \times 10^{19}$ & 1.54 & 0.6
\end{tabular}

The equilibrium reconstructions provide adequate profile information for the modeling of beam deposition with the BEAMS3D code. Table 3 depicts key quantities for the reconstructed time slices. In general, the plasma betas and currents were small 

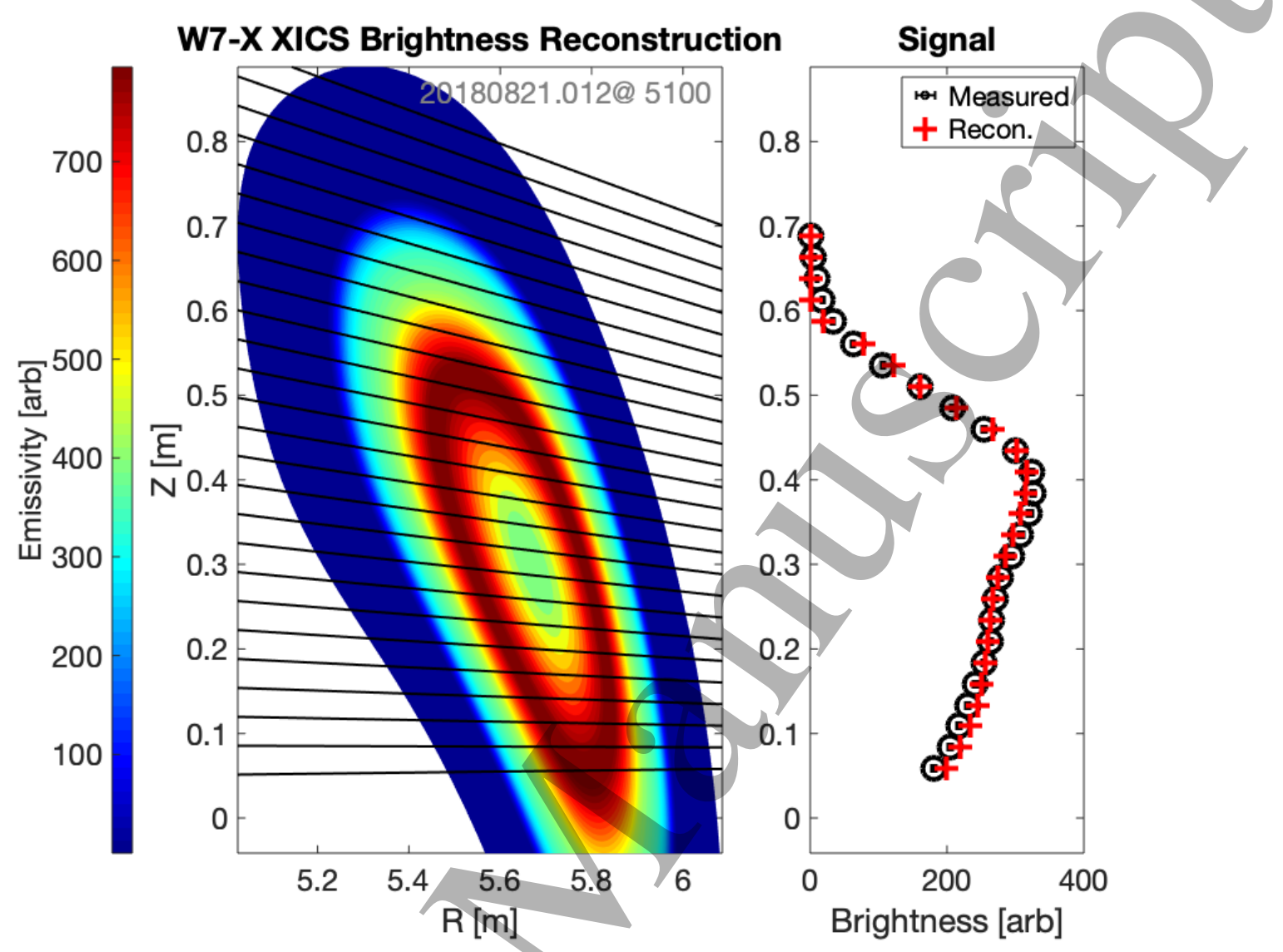

Figure 9. Reconstructed XICS Brightness data (right) depicted against a rendering of the effective emissivity profile in the XICS toroidal plane for the standard configuration medium density discharge (20180821.12).

and played only a modest role in the reconstructions (through shifts in the core flux surfaces). In the following subsections, the medium density standard configuration discharge (20180821.12) will be used as an example of the reconstructed parameters.

\subsection{XICS Effective Emissivity}

The forward model of the XICS data requires that the effective emissivity be reconstructed. The yalue of this effective emissivity can be related back to the density of the Argon charge states, but this is out of the scope of this paper. It should also be noted that the effective emissivity does not directly influence the equilibrium calculation itself, but rather just the forward modeling of the XICS ion and electron temperature measurements. Figure 9 depicts a stereotypical reconstructed emissivity mapped into the toroidal plane of the XICS system. A hollow emissivity profile provides an excellent fit to the measured brightness from the XICS system. It has been suggested that the slight up-down asymmetry seen in the core region of the brightness could be addressed through variation of the brightness along a flux surface. 
W7-X BEAMS3D Validation

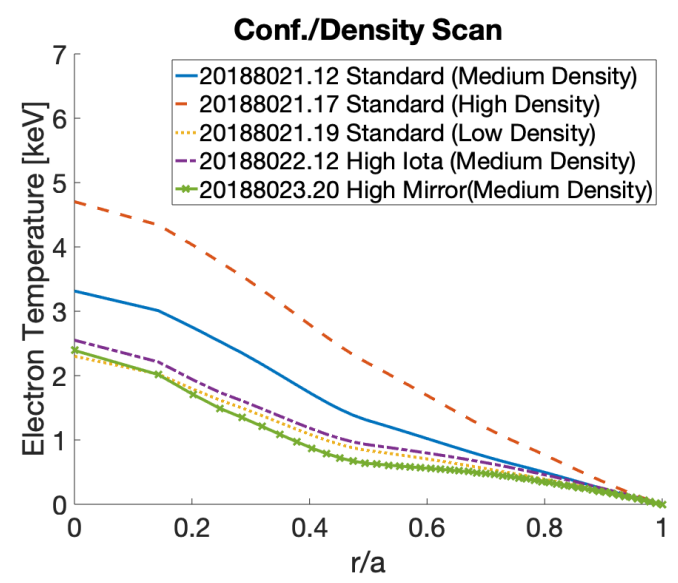

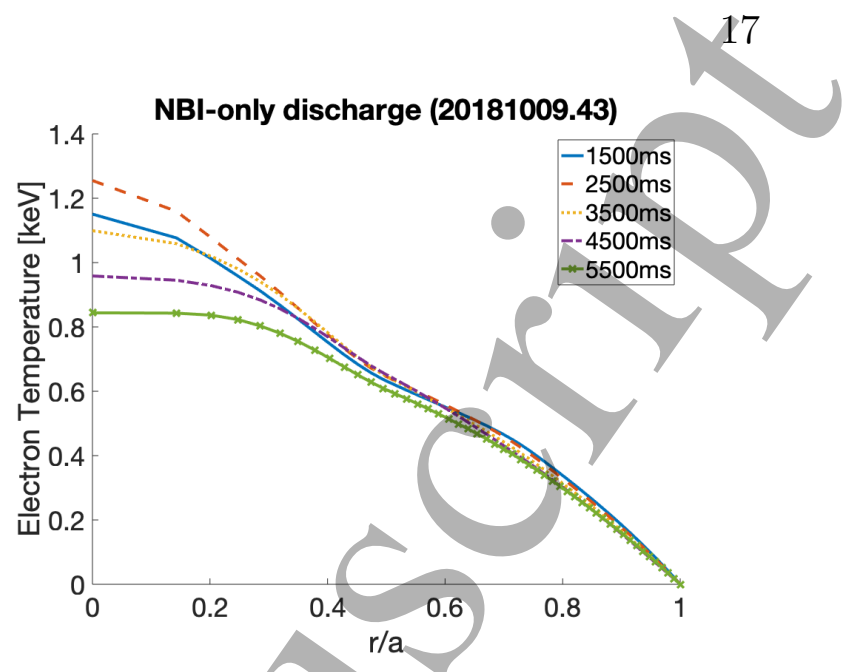

Figure 10. Reconstructed electron temperature profiles for the density/configuration scan (left) and the pure NBI discharge (right).

\subsection{Electron Temperature Profiles}

Of the discharges considered, the electron temperature was well diagnosed by Thomson scattering, ECE, and XICS. The exception being the NBI only discharge (20181009.43) which achieved densities above the X2 emission cutoff, and had poor signal for the XICS system (due to insufficient Argon density). In that discharge, only Thomson was available for electron temperature data. Figure 10 depicts the reconstructed temperature profiles for the discharges. The high density standard configuration discharge exhibits a higher temperature due to the necessity of higher ECRH power at that density. Differences in electron temperature may also be attributed to differences in ECRH power between discharges.

Figure 11 depicts the forward model of the Thomson electron temperature based on the reconstructed electron temperature profile. The magnetic axis location (depicted by the dashed line) agrees well with the peak in the measured electron temperature. In the edge regions the model agrees well with measurements. The reconstructed core electron temperature appears slightly cooler than that measured by Thomson. Given the magnitude of the error bars, this slight disagreement is reasonable. It is mostly likely due to the ECE and XICS measurements suggesting a slightly smaller value of core electron temperature. Large error bars in the plot for channels on the lefthand side of the plot can be attributed to laser stability and calibration issues.

Figure 12 depicts the forward model of the ECE emission for this medium density standard configuration discharge. Here the TRAVIS code is used to provide a forward model. In general a good agreement is found between the measured and reconstructed ECE signal. It should be noted that good agreement is only found if the optimizer is allowed to vary the magnetic field strength (through the enclosed toroidal flux variable, PHIEDGE). This is because ECE provides a mapping between electron temperature and the magnetic field strength (in the simplest approximation). This as opposed to Thomson which provides a mapping between electron temperature and real space. 
W'-X BEAMS3D Validation 18

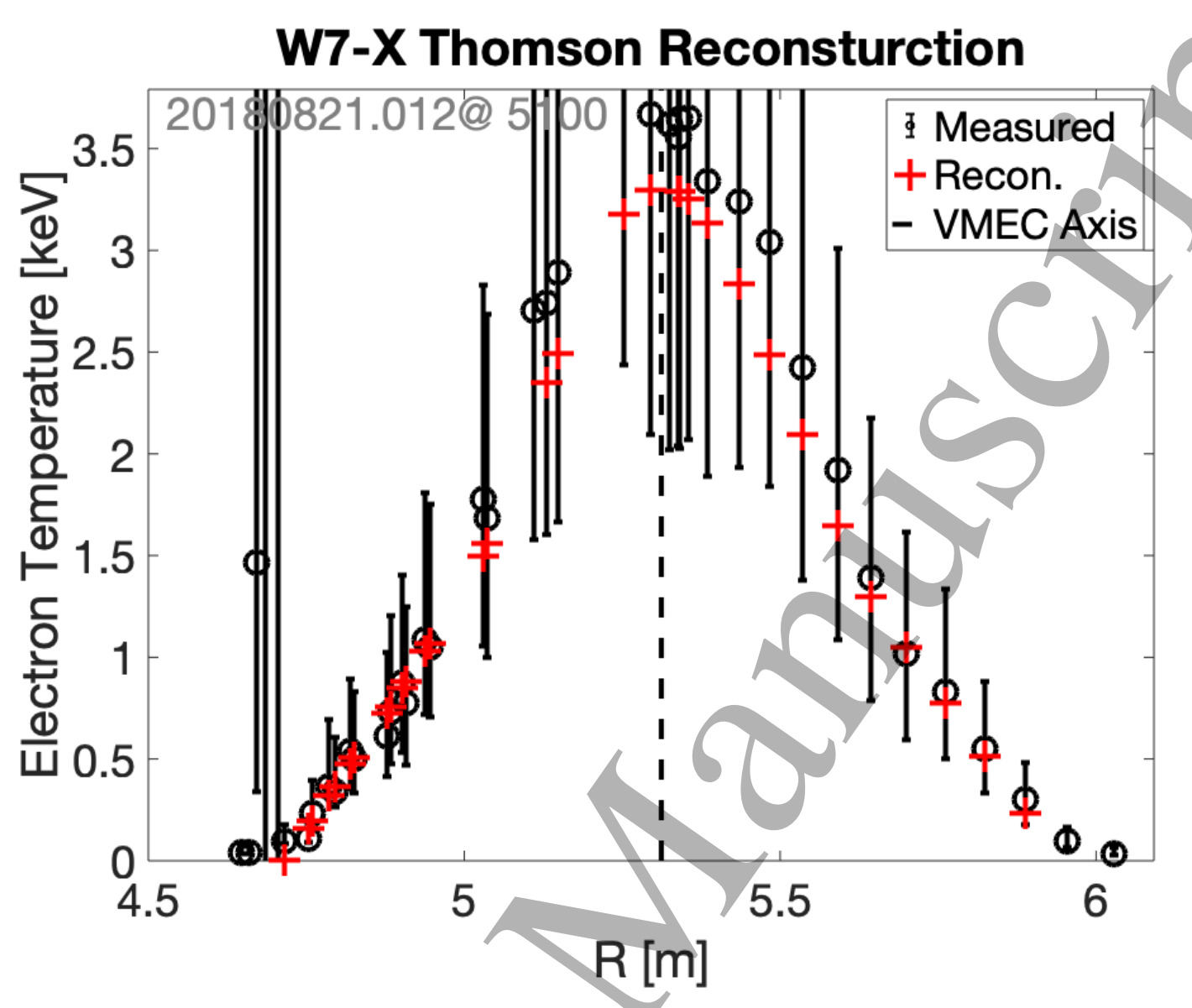

Figure 11. Reconstructed Thomson data for the medium density standard configuration discharge (20180821.12). Not all data points were considered in the reconstructions, only those whose forward model is depicted.

Reconstructing with both signals provides a strong constraint on the equilibrium. It should also be noted that the electron density enters into this calculation as well. Finally, both the $\mathrm{X}$ and O-mode contributions were included in the reconstruction in order to get good agreement on the low field (low frequency) side of the spectrum.

The XICS system also provides a measurement of the electron temperature through the measurement of satellite lines. Figure 13 shows an example of such a reconstruction. In general, good agreement is found for most channels. In the edge it is clear that the signal is low which which is not to say that the electron temperature is low, just that as the electron temperature drops below $1 \mathrm{keV}$ the signal becomes vanishingly small. It should also be noted that the peak in this XICS measurement does not correlate with a peak in the electron density. It would appear that path length through the plasma plays a major role in the signal along with the peak in XICS brightness. This highlights the need for performing reconstructions when interpreting data such as this. 


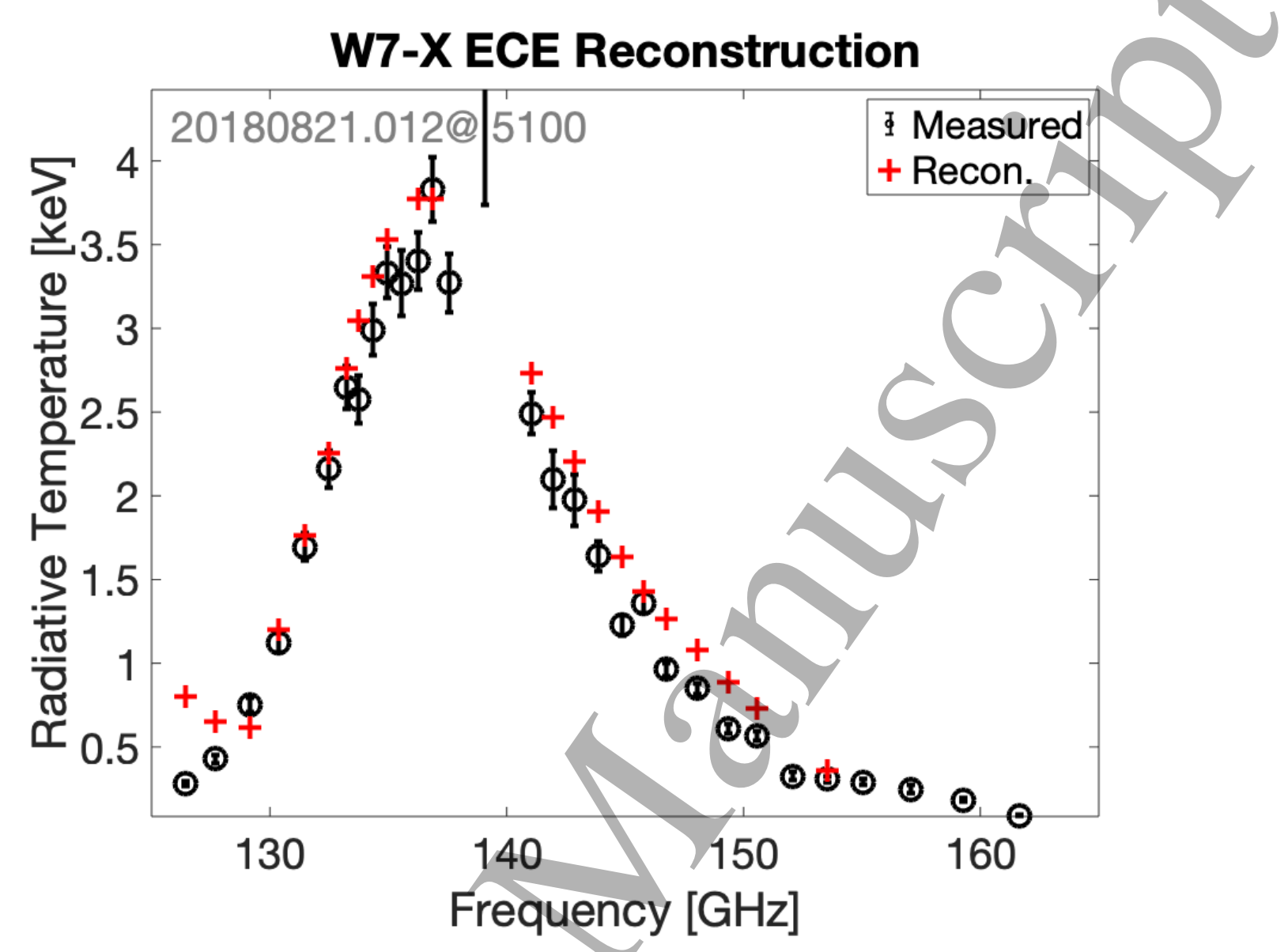

Figure 12. Reconstructed ECE data for the medium density standard configuration discharge (20180821.12). Not all data points were considered in the reconstructions, only those whose forward model is depicted.

\subsection{Ion Temperature Profiles}

The ion temperature in these discharges was measured by both XICS and CXRS systems. Figure 14 shows the reconstructed ion temperature profiles for the discharges and timeslices considered in this work. The discharges in which NBI was fired into an ECRH plasma show relatively consistent profiles with little appreciable variation in ion temperature. This appéars consistent with the current understanding of ion heat transport in W7-X [29]. The pure NBI discharge shows a general trend of decreasing ion temperature as the discharge evolves. This appears consistent with a plasma which has an ever increasing density at fixed injected power.

Figure 15 shows the reconstructed and measured CXRS data for a stereotypical discharge. The agreement here is adequate for the purposes of neutral beam deposition modeling (as will be shown in the next section), as the deposition is only weakly dependent on the ion temperature. Scatter in the dataset can be linked to possible nonlocality in the measurements. Specifically, we are treating the CXRS measurements as point measurements at which the ion temperature is measured. But the actual system integrates over the width of the neutral beam line. 

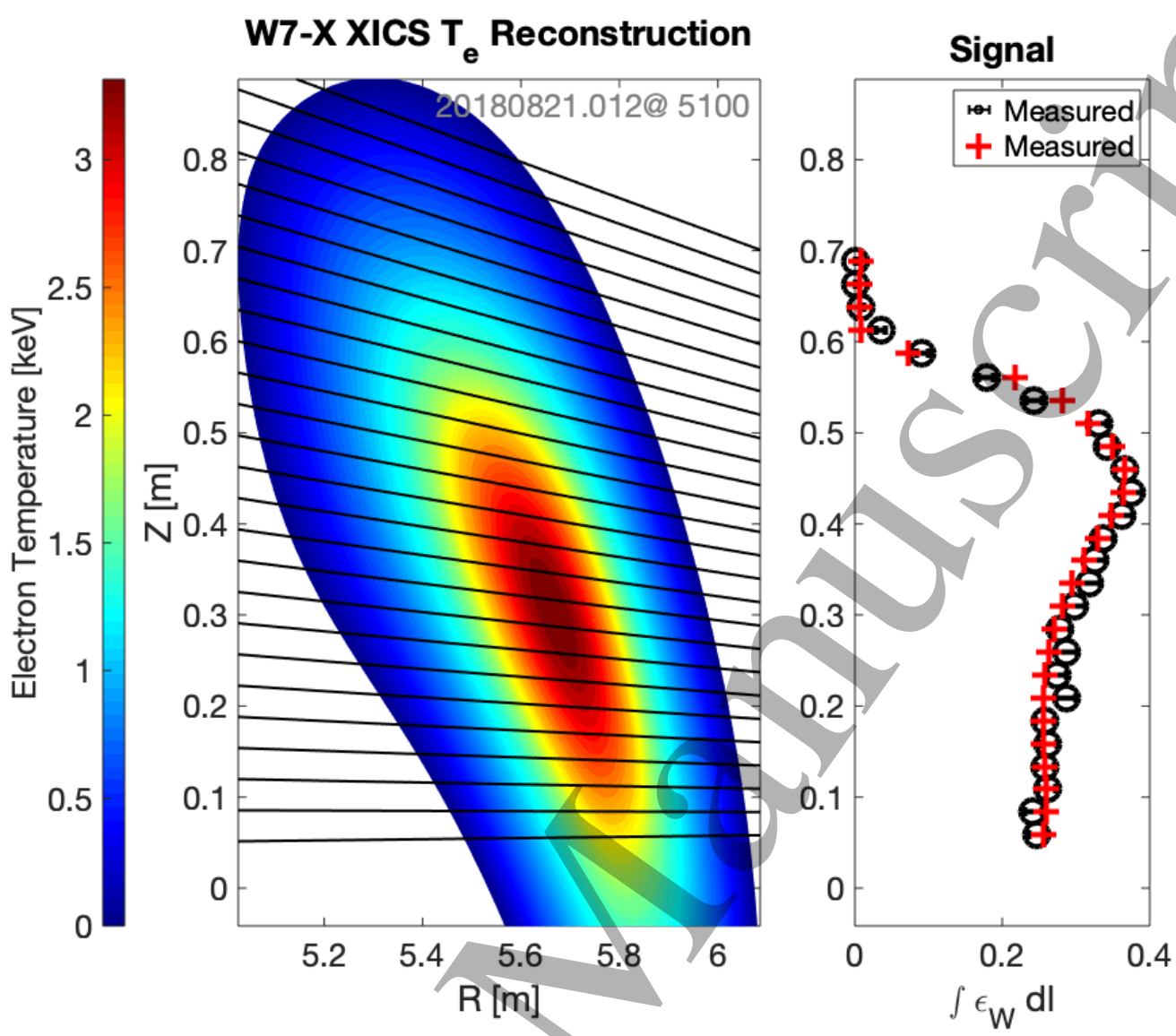

Figure 13. Reconstructed XICS electron temperature data for the medium density standard configuration discharge (20180821.12).
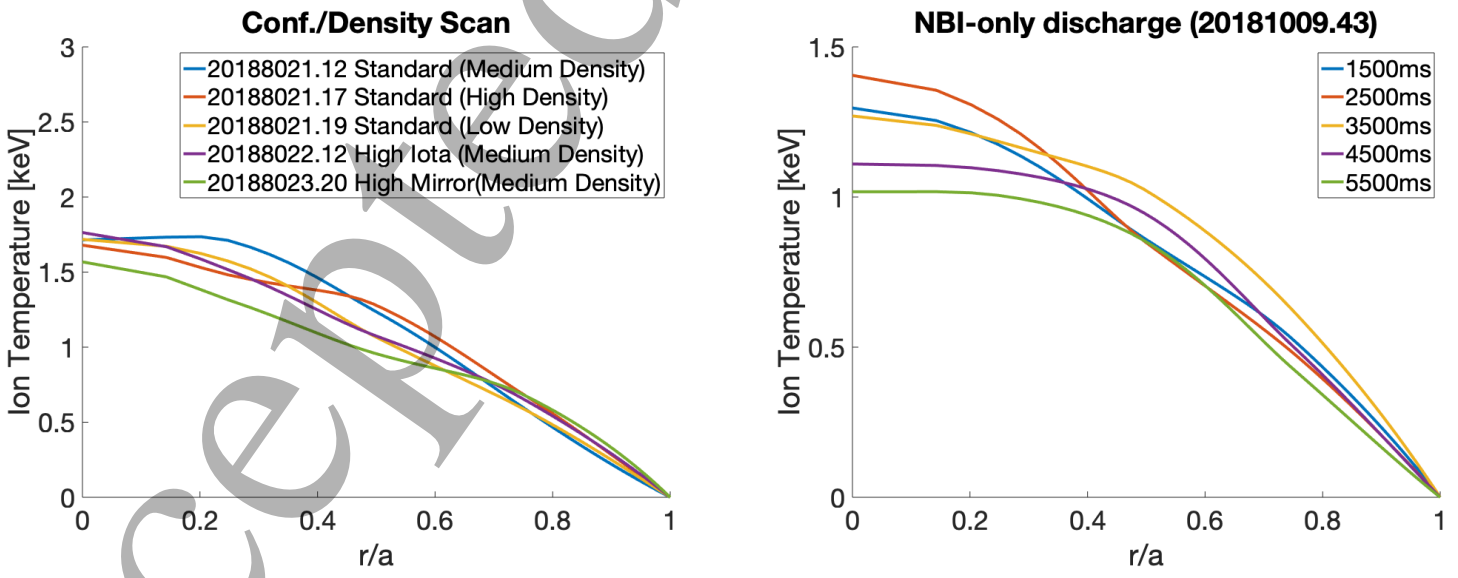

Figure 14. Reconstructed ion temperature profiles for the density/configuration scan (left) and the pure NBI discharge (right).

The agreement between reconstructed and measured XICS signals appears very good. Figure 16 shows the detail of this agreement. It should be noted that because the XICS brightness in the edge channels is low, the reconstructed temperature profile 


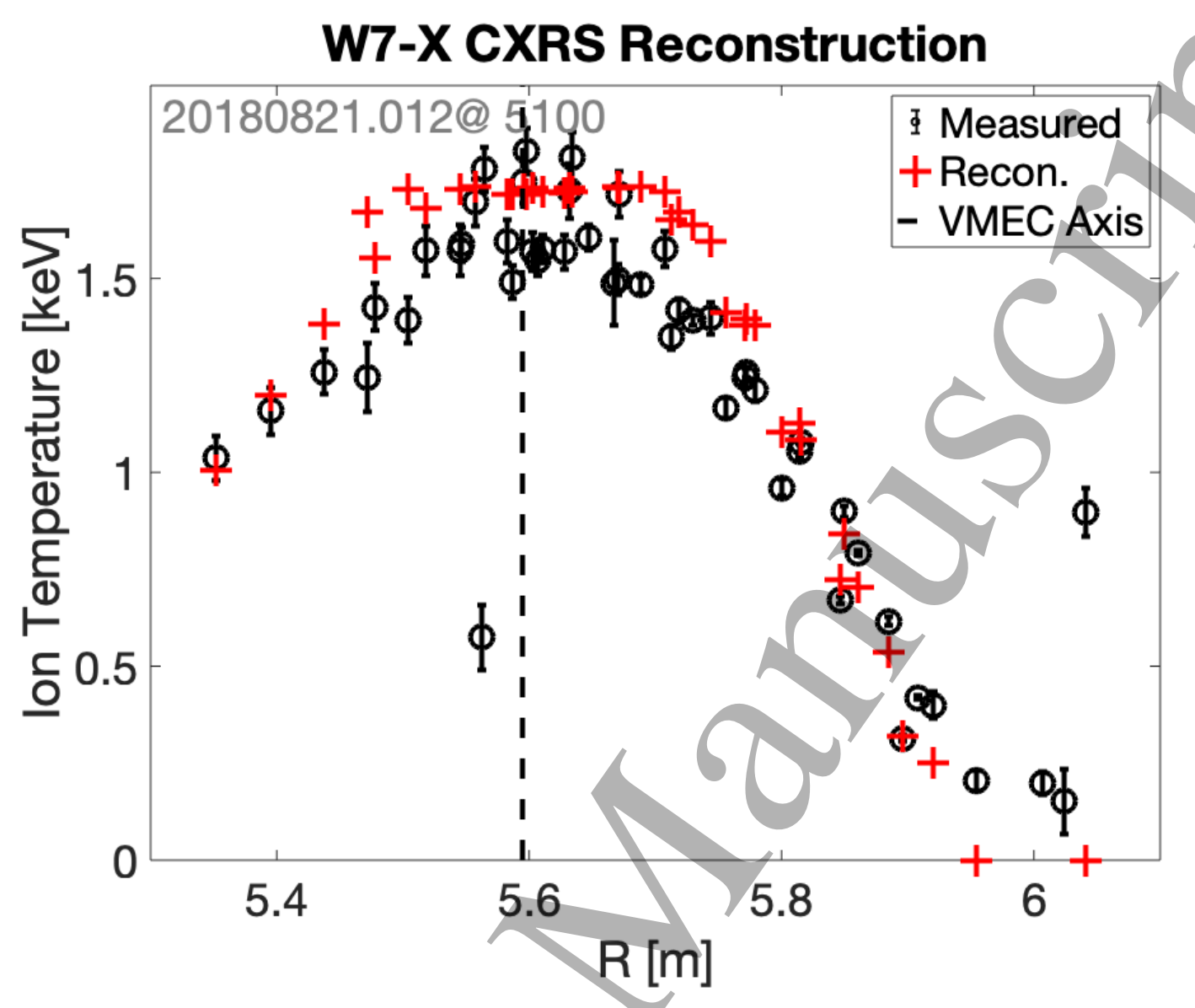

Figure 15. Reconstructed CXRS ion temperature data for the medium density standard configuration discharge (20180821.12).

is not strongly determined in the edge. For this discharge a slight hollowness in the very core of the plasma is seen. Given the scatter in the CXRS data and good fit to the XICS this may be evidence of off-axis heating due to beam deposition. Such analysis is left to future work.

\subsection{Electron Density Profiles}

The electron density profiles for the ECRH discharges are remarkably similar in shape while the NBI only discharge shows a clear peaking in the density profile (figure 17). The discharges with ECRH have flat density profiles inside of $r / a=0.5$ which is consistent with the majority of non-pellet fueled discharges in W7-X. Meanwhile the NBI only discharge shows a strong peaking of the density profile inside of $r / a=0.5$ and almost no-change in the density profile outside this region.

The reconstructed Thomson data is shown in figure 18, along with the reconstructed line integrated density and effective ion charge number $\left(Z_{\text {eff }}\right)$. The fit to the data appears adequate given the scatter in the datapoint for the discharge. During the reconstruction process STELLOPT normalizes the Thomson data when comparing the 

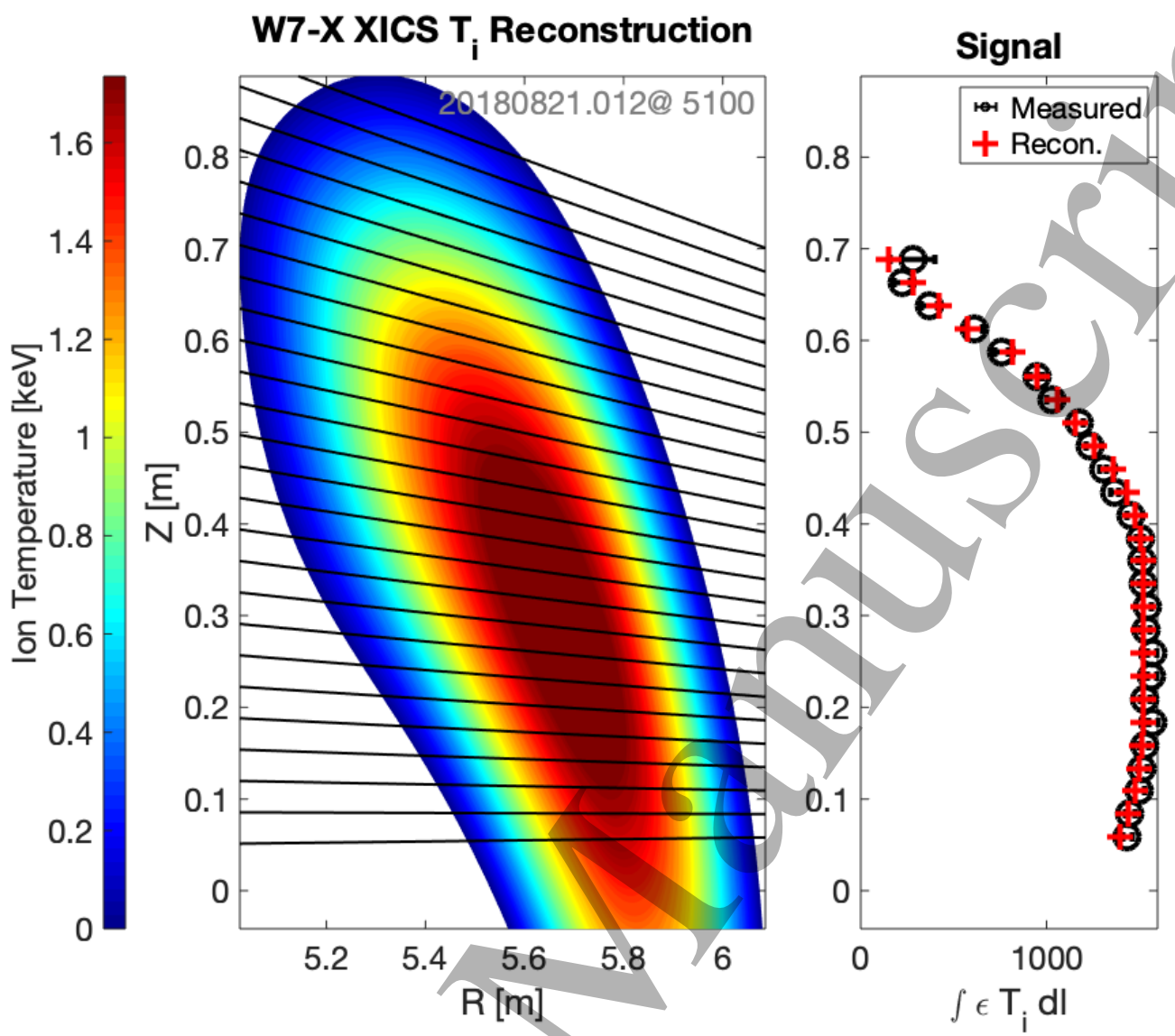

Figure 16. Reconstructed XICS ion temperature data for the medium density standard configuration discharge (20180821.12).
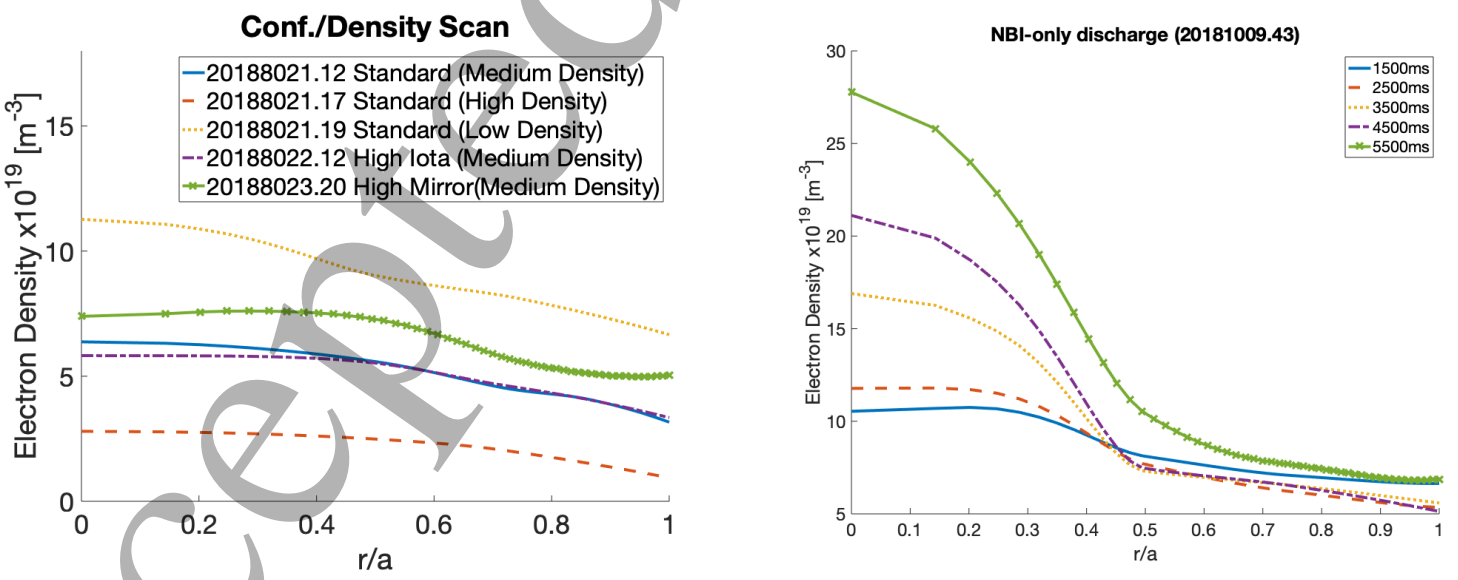

Figure 17. Reconstructed electron density profiles for the density/configuration scan (left) and the pure NBI discharge (right).

synthetic diagnostic response to the measured value. This allows the Thomson data to provide feedback on the profile shape without influencing the amplitude information. The amplitude of the density profile is determined through fitting of the line integrated 


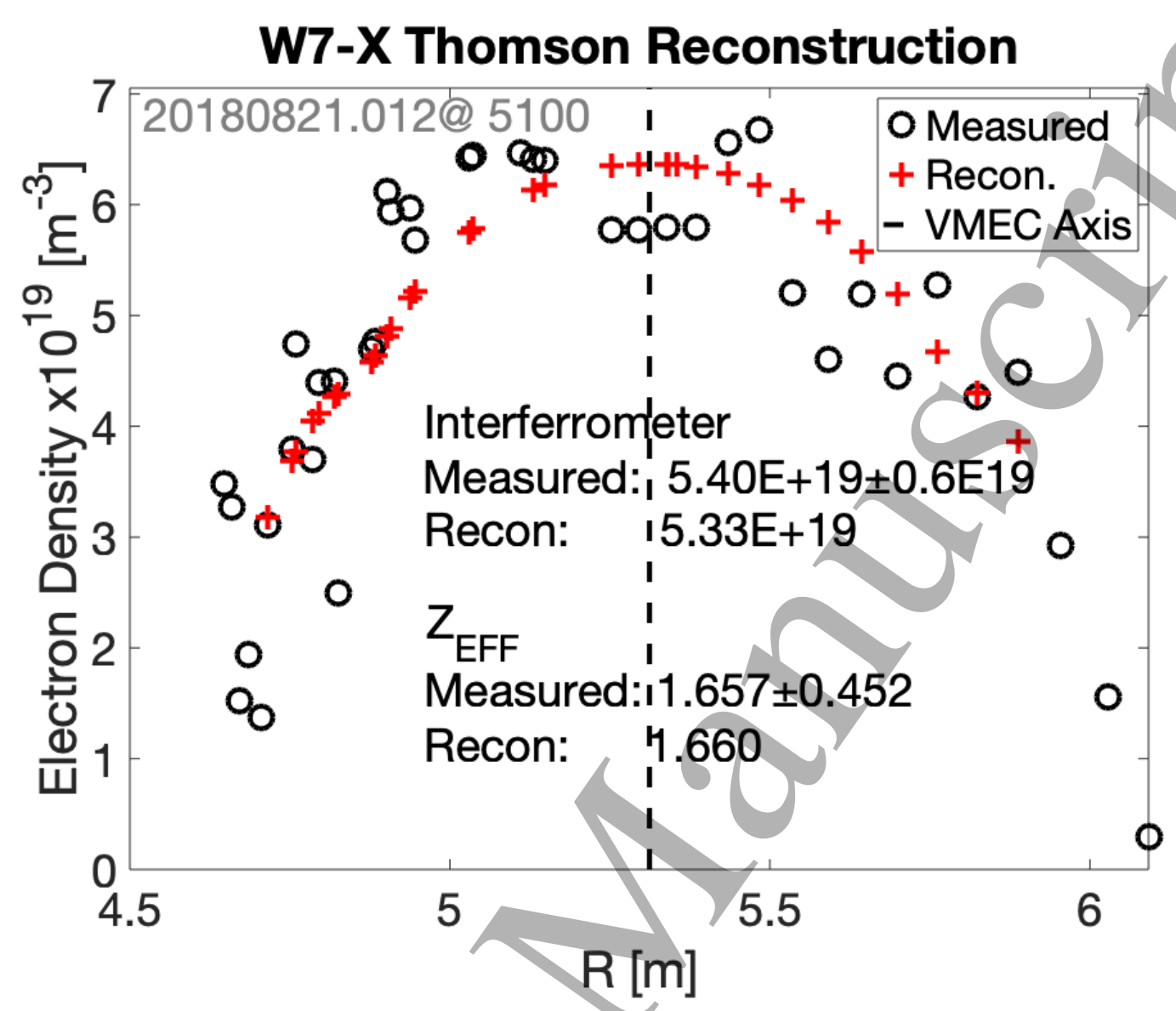

Figure 18. Reconstructed Thomson electron density data for the medium density standard configuration discharge (20180821.12). The measured and reconstructed line integrated density and $Z_{\text {effective }}$ have been displayed as well, showing good agreement.

electron density. Here we see that the fit to the measured data is in excellent agreement. The line integrated effective ion charge number is also found to be a good match to measurement.

\subsection{Current Density Profiles}

The reconstruction of the equilibrium current density profiles can be seen in figure 19. At medium to high density all configurations show bootstrap-like current density profile with small amounts of edge current density. The major departure being the low density discharge which indicates some evidence of larger edge current densities. A reversal in current direction in the high iota configuration is present as predicted by neoclassical theory. For the pure-NBI discharge, most time slices show a bootstrap-like profile with some departures seen at $3500 \mathrm{~ms}$ and $5500 \mathrm{~ms}$. In general the currents are small, implying that variations in the current density have negligible effect on the rotational transform or equilibrium properties in general. This robustness of magnetic configuration to finite plasma beta is a feature of the design of W7-X.

Figure 20 depicts the forward modeled magnetics signals as compared to the 

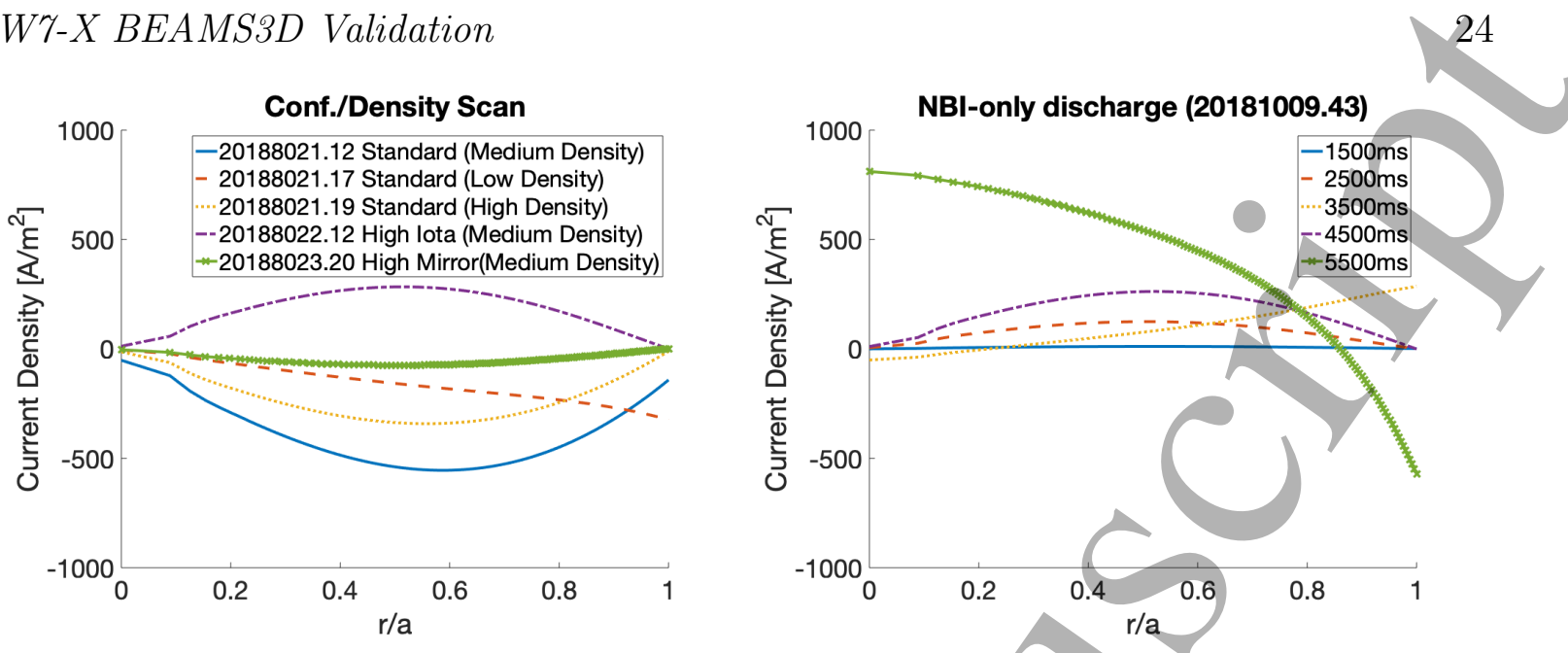

Figure 19. Reconstructed current density profiles for the density/configuration scan (left) and the pure NBI discharge (right).

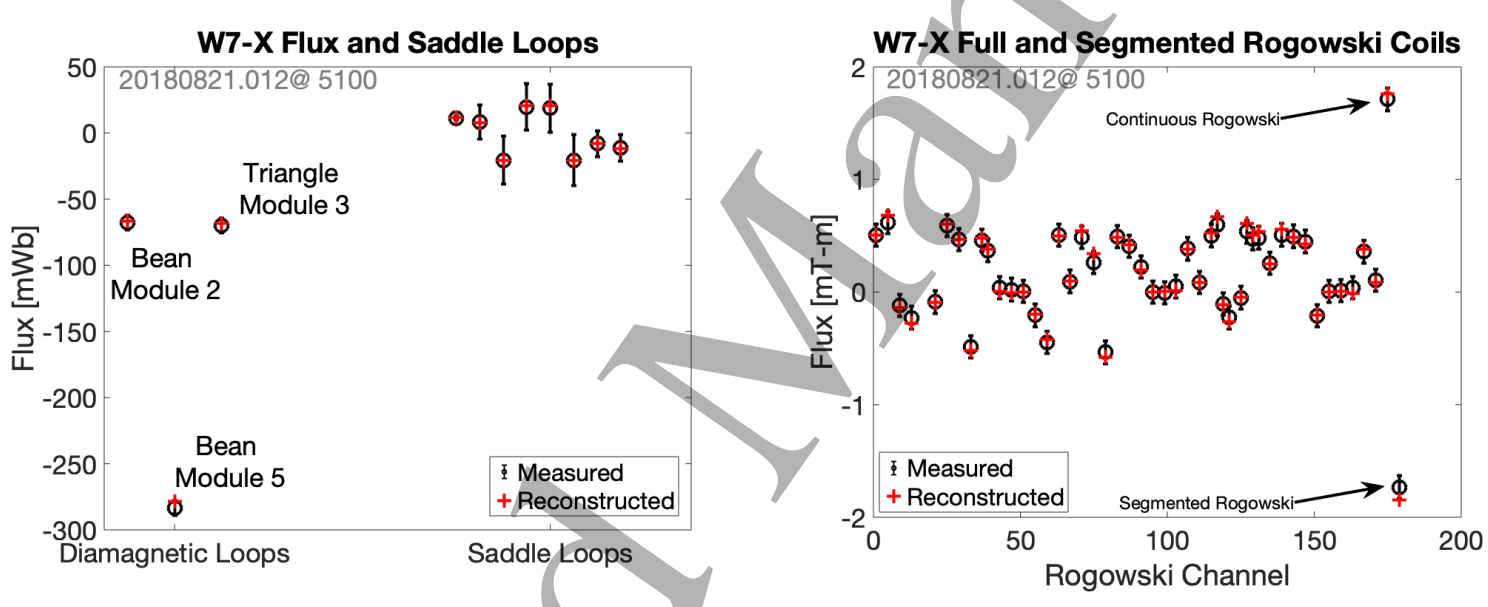

Figure 20. Reconstructed fluxloop (left) and Rogowski signals (right) for the medium density standard configuration discharge (20180821.12).

measured values. Good agreement is seen for both the diamagnetic loops in the triangular and bean shaped cross sections. In addition, the saddle loops show good agreement as well. As our equilibrium model is stellarator symmetric only one set of 8 saddle loops in one module is simulated. An average of similar saddle loops in different modules is used to weight the reconstruction parameters. This attempts to account for any symmetry-breaking fields that may be present. The segmented Rogowski coils show similar levels of agreement with the full Rogowski coils showing the greatest discrepancy. It should be noted that for this reconstruction a pressure scaling factor of 1.08 was reconstructed. This would suggest a non-thermal contribution to the plasma pressure of around $1 k J$ in terms of stored energy, presumably from the beam-ions. 

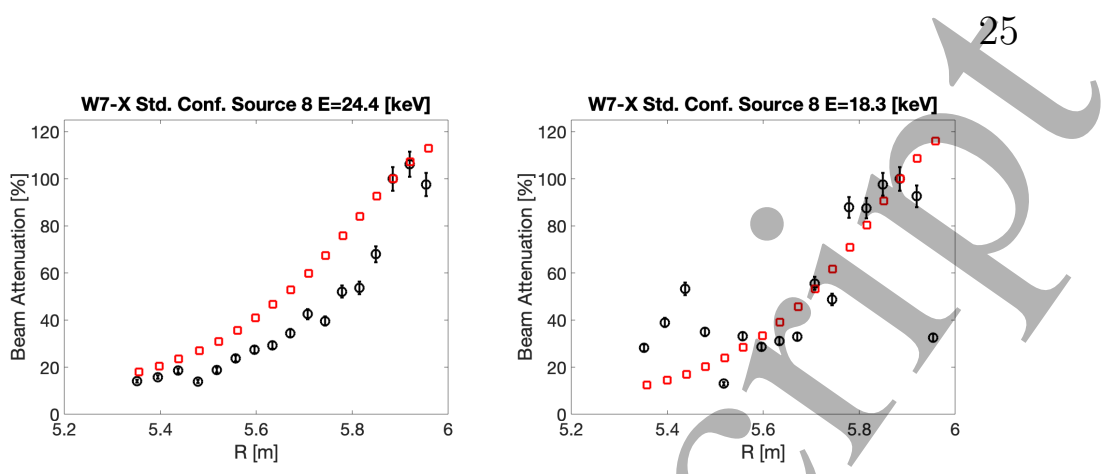

Figure 21. Neutral beam attenuation for the full, half, and third energy component for a single source discharge (20180821.12). The neutral beam fires from right to left in these plots. Beam attenuation is taken as relative measure based on third channel at $R \sim 5.9 \mathrm{~m}$.

\section{Beam Deposition}

Using the reconstructed equilibria and profile information, beam deposition calculations were performed using the BEAMS3D code and compared to the measured beam attenuation. Comparisons with measured neutral beam attenuation are used to confirm the accuracy of the beam model. The effect of the uncertainties in the reconstructed profiles on the simulated results was also assessed. Plasma density was found to play the dominant role in the simulated beam attenuation profile.

Figure 21 compares the measured and simulated neutral beam attenuation for a discharge (20180821.12) in which a single source (S8) was used. The agreement between the measured and simulated data appears adequate, given the scatter in the measured data. The simulated results show a clear attenuation of the beam as it penetrates into the plasma (firing from right to left in the plots). The measured data shows some features, such as increasing attenuation, which are unphysical. The presence of these features can be explained by how the measured data is interpreted as attenuation. Each datapoint in the plot corresponds to a channel of spectroscopic measurement viewing across the width of the beam-plasma interaction region. In order to infer a beam density from this data, the ion temperature and electron density are needed. These values are then used to calculated beam stopping coefficients using the ADAS database (for $H^{0}$ ). From the density and these coefficients, a beam attenuation can be calculated. The deviation from a monotonically decreasing profile (right to left) can be considered an additional error bar on the measured data.

As the profile information enters into both the simulated data and interpretation of the measured beam attenuation the effect was assessed. In general it was found that the simulated data showed little sensitivity to either electron temperature or ion temperature variations, while variations in electron density have a strong effect. Figure 22 depicts the effect of a $\pm 10 \%$ density variation on the full energy component of discharge 20180821.12. Clearly the simulated data shows the greatest sensitivity to changes in the electron density. The effect on the measured data is small as the ADAS beam stopping coefficients are not strongly dependent on the electron density in these 
W'-X BEAMS3D Validation 26

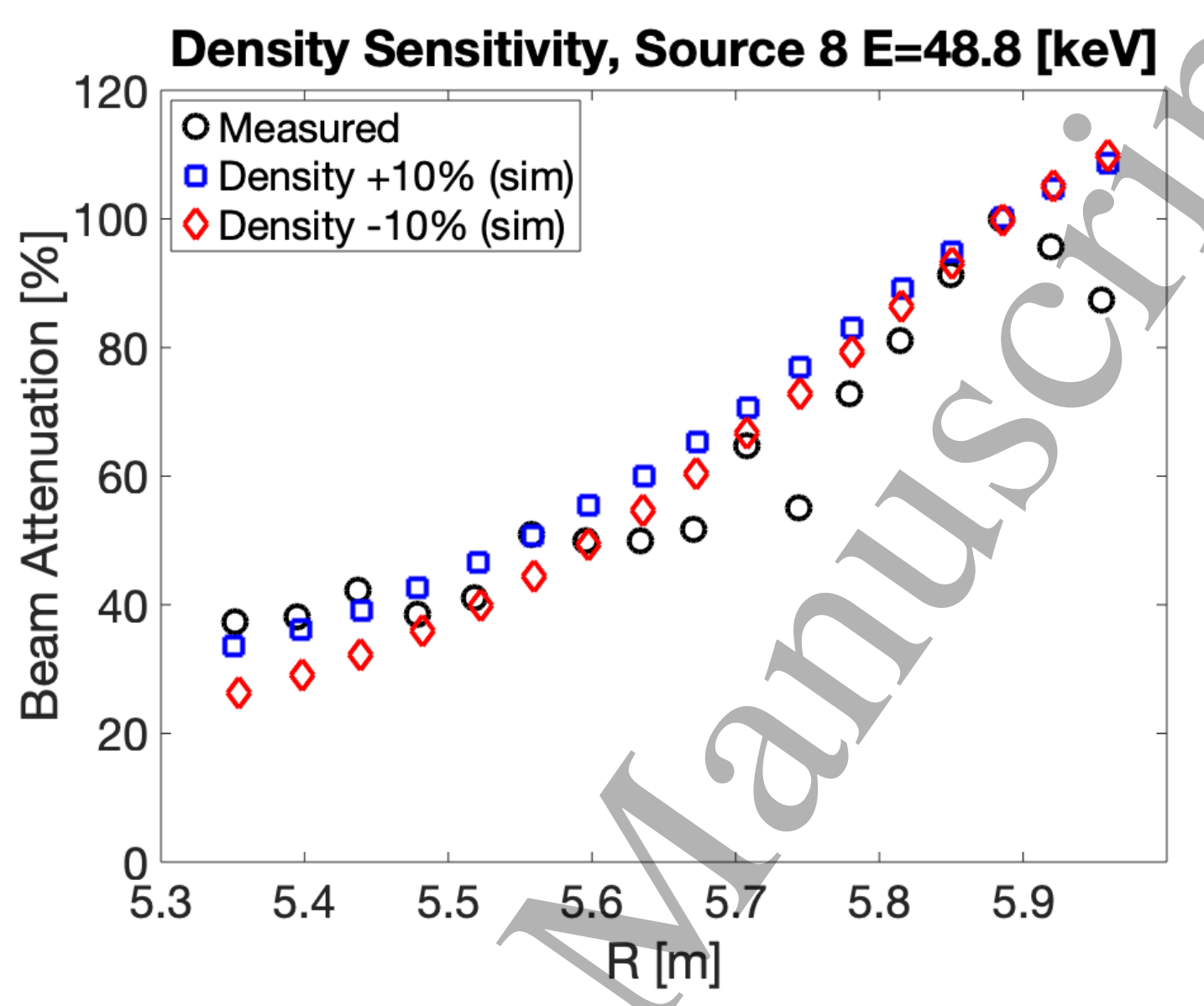

Figure 22. Effect of electron density on simulated beam attenuation (squares) as compared to the measured data (circles) for the full energy component of the beam. Beam attenuation is taken as relative measure based on third channel at $R \sim 5.9 \mathrm{~m}$.

density regimes. This variation in density has the strongest impact on the value of the total beam attenuation (lefthand most data points in figure 22).

Variation in magnetic configuration plays little role in beam attenuation as shown in figure 23. This lack of variation is attributed to the fact that similar plasma profile shapes are obtained in these discharges and the fact that changes in the beam-plasma path length are only slightly different between these configurations. The birth location in physical space is largely unchanged in these configurations as are the pitch angles which are populated by the beams. The variation in total attenuation of the beam is attributed to differences in the total shine through between discharges (variation in target plasma density).

Variations in target plasma density are investigated in figure 24. In these discharges the density profiles are similar despite large variations in the target density and the magnetic configuration is held fixed (standard configuration). The general shape of the beam attenuation curve is rather similar between these discharges while the magnitude of the attenuation clearly increases with increasing plasma density. This suggests that the shape of the attenuation curve is not a strong function of total plasma density. It 


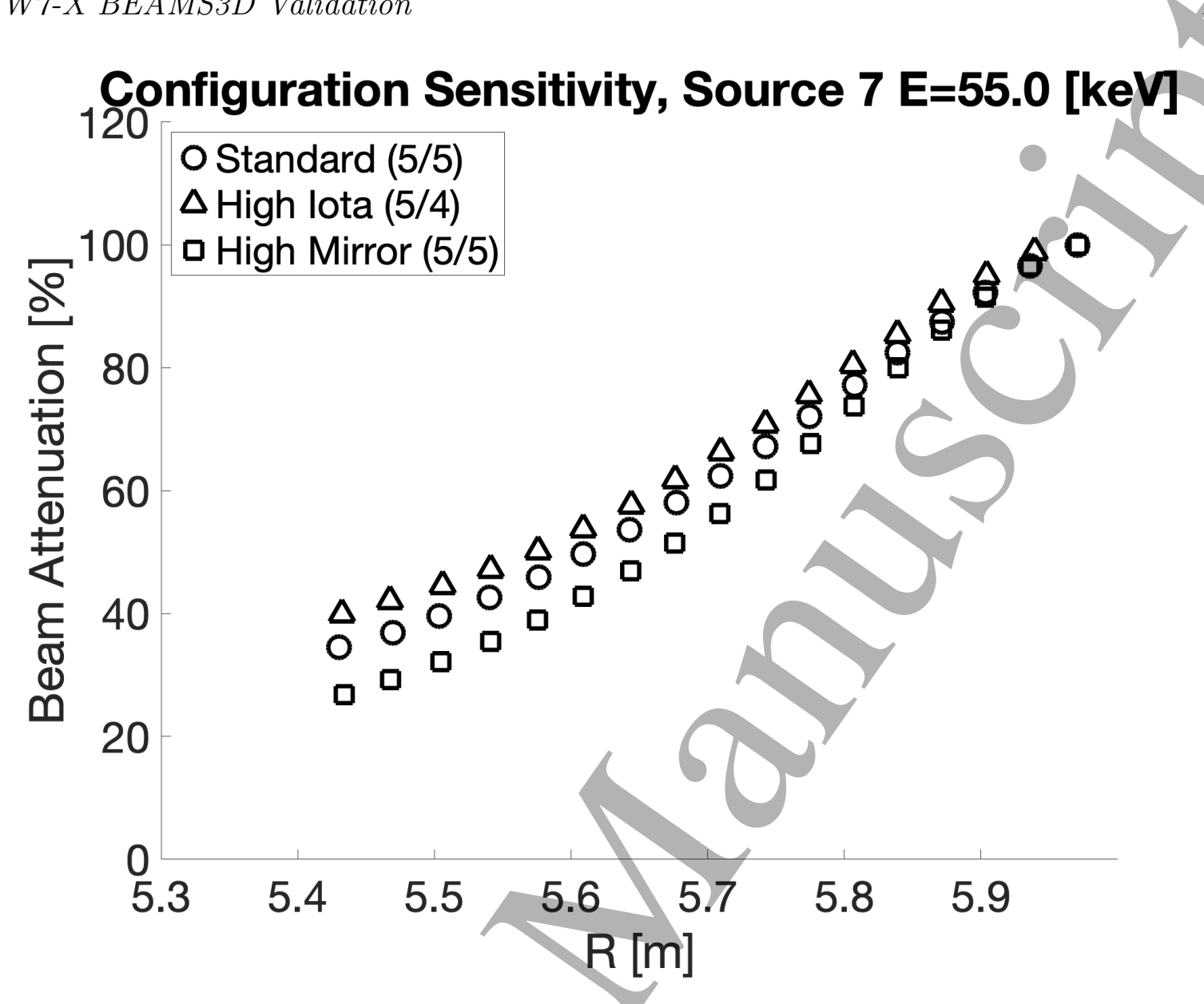

Figure 23. Simulated neutral beam attenuation of source 7 for the standard (20180821.012), high iota (20180822.12), and high mirror (20180823.20) magnetic configurations at medium densities $\left(\sim 5-8 \times 10^{19}\left[\mathrm{~m}^{-3}\right]\right)$. The variation in beam attenuation can be explained by variances in the target plasma density between discharges. The legend indicates the edge rotational transform resonance for each of these configurations. Beam attenuation is taken as relative measure based on first channel at $R \sim 5.95 \mathrm{~m}$ in this plot.

does however indicate, as is expected, that the total attenuation is a strong function of the plasma density. Sensitivity studies confirm this behavior, and suggest that the shape of the attenuation profile is influenced by the density profile shape. Measured beam attenuation is only available for discharge 20180821.12 for these discharge sets.

A discharge in which ECRH is used to startup a plasma, then turned off, provides a scan of the effect of pure NBI heating along with changes in the magnitude and shape of the density profile. In figure 25, comparisons between the simulated and measured beam attenuation are plotted at three time-slices. The lefthand most plot is from $500 \mathrm{~ms}$ after ECRH has been switch off, and has a density profile similar to that of discharge 20180821.19. The density profile is fairly flat and good agreement is found between the simulated and measured results. The middle most plot is taken $2500 \mathrm{~ms}$ into the pure NBI phase of the discharge, where the density is showing strong core peaking. The attenuation profile is becoming more steep with more particles being attenuated at the 


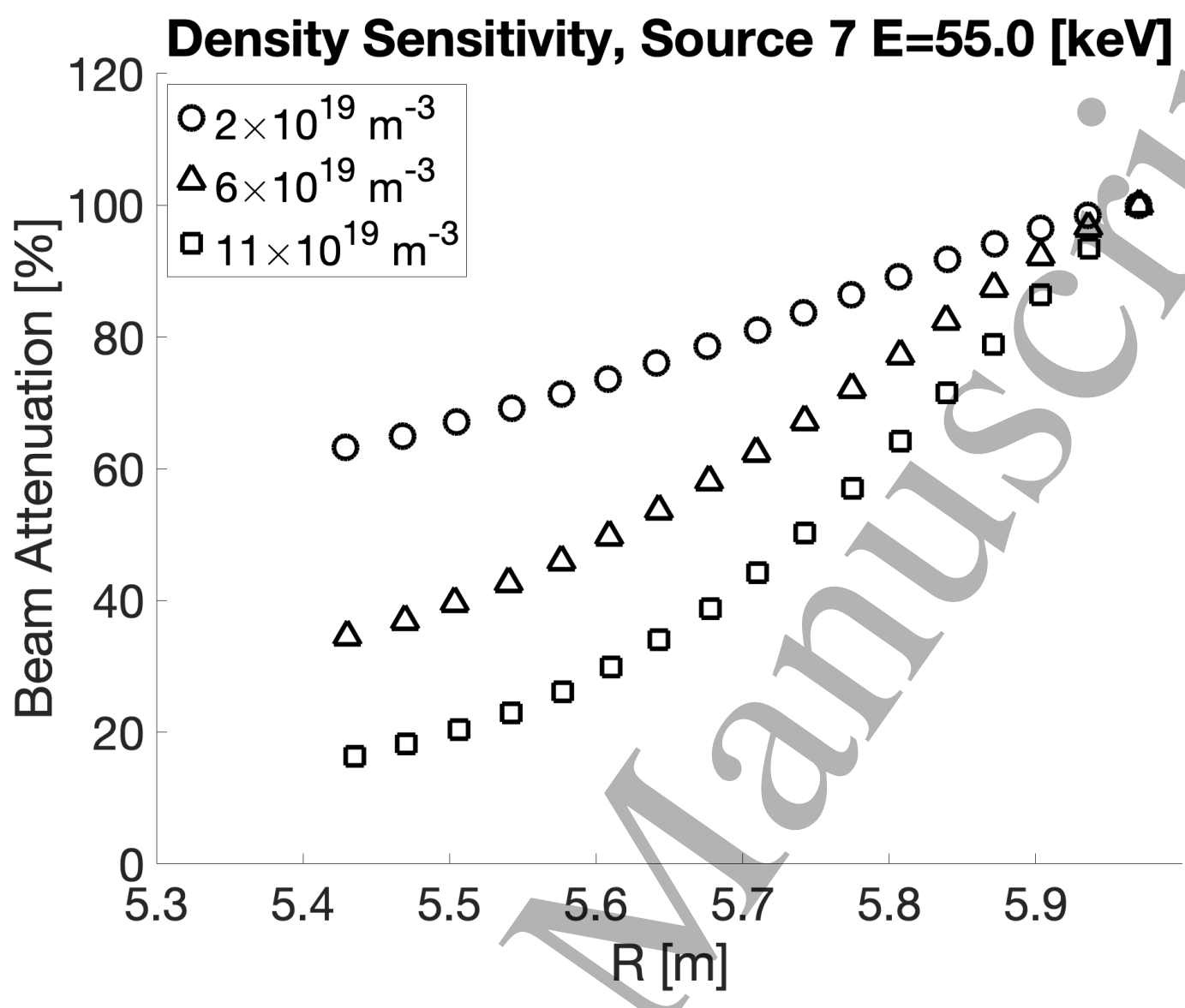

Figure 24. Simulated neutral beam attenuation of source 7 for the standard magnetic configuration at low density (20180821.017), medium density (20180821.12), and high density (20180821.19). In addition to the total beam attenuation, the shape of the attenuation curves varies between discharges as more of the beam is attenuated sooner in the plasma. Attenuation is relative to the first channel at $R \sim 5.95 \mathrm{~m}$.
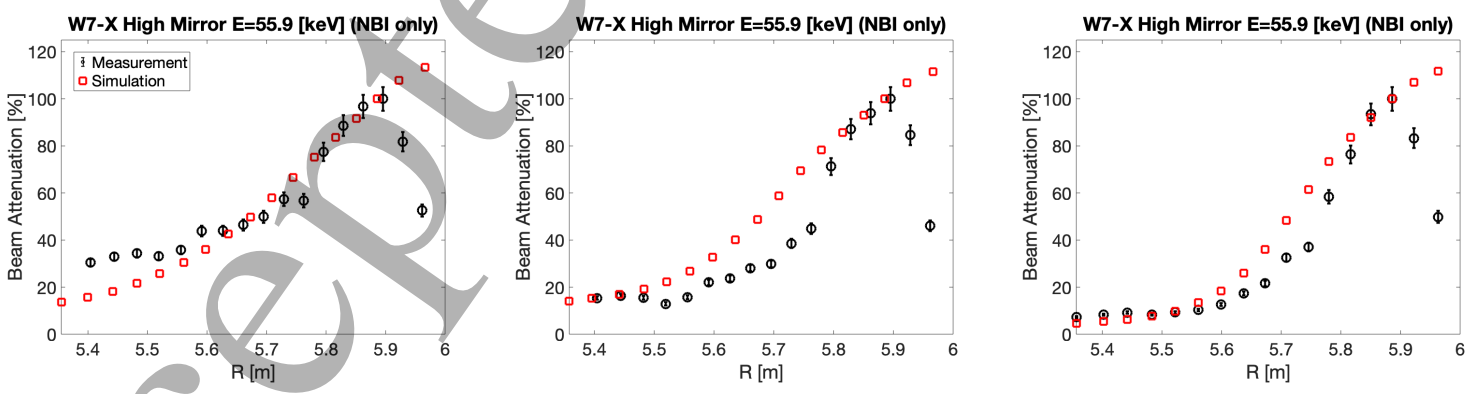

Figure 25. Neutral beam attenuation for the pure-NBI discharge (20181009.43) at $1500 \mathrm{~ms}$ (left), $3500 \mathrm{~ms}$ (middle) and $5500 \mathrm{~ms}$ (right) time slices. The full energy component of source 8 is shown in these plots. Beam attenuation is taken as relative measure based on third channel at $R \sim 5.9 \mathrm{~m}$.

outboard side of the plasma (right hand side of the plot). In the righthand most plot the beam is being strongly attenuated as densities in the core are becoming strongly peaked. It should be noted that outside of $r / a=0.5$ the density profiles between time-slices are 
similar in shape and magnitude, despite the large change in core density. The good agreement between simulations and measured data suggest that the BEAMS3D code is accurately capturing the physics of the beam-plasma interaction.

\section{Conclusions}

Experimental data from the Wendelstein 7-X experiment was used to reconstruct equilibrium and kinetic profile data, allowing modeling and validation of the neutral beam deposition in the BEAMS3D code. A tool for rapid profile fitting of W7-X data has been introduced (WAPID_FIT). Upgrades to the STELLOPT code allowed it to reconstruct kinetic profiles using multiple diagnostics. Specifically, the XICS and ECE diagnostics have now been included. Simulations of neutral beam deposition with BEAMS3D, using these equilibria and profiles, were then compared to measurements of beam attenuation. Good agreement was found over a wide range of relevant operational parameters. These simulations provide the initial conditions for simulations of fast ion losses. Validated deposition profiles thus provide a basis for future work validating wall loss and fast ion confinement calculations in the future.

In general, it was found that the electron density played the largest role in neutral beam attenuation (as compared to ion temperature, electron temperature, and effective ion charge). As simulations of fast ion dynamics depend on the sources of fast-ions, accurate measurement of electron density is a key to performing such simulations moving forward. The reconstructions and deposition calculations performed in this work will serve as inputs for those future calculations.

In this work a forward model of beam attenuation was developed using the data from the BEAMS3D simulations. In assessing this forward model it was found that the number of particles tracked could be significantly reduced without a significant reduction in accuracy. This reduces the runtime for a single calculation and the computational load. It has been suggested that this forward model could be extended to include a synthetic charge exchange measurement, and subsequently be included in the STELLOPT reconstructions. This would then allow the beam width through the plasma to be taken into account. Currently the charge exchange measurements are treated as point sources of $T_{i}$ in STELLOPT. The development and implementation of such work is left to the future.

\section{Acknowledgments}

This work has been carried out within the framework of the EUROfusion Consortium and has received funding from the Euratom research and training programme 2014-2018 and 2019-2020 under grant agreement No 633053. The views and opinions expressed herein do not necessarily reflect those of the European Commission.

[1] T. Klinger, T. Andreeva, S. Bozhenkov, C. Brandt, R. Burhenn, B. Buttenschön, G. Fuchert, B. Geiger, O. Grulke, H.P. Laqua, N. Pablant, K. Rahbarnia, T. Stange, A. von Stechow, N. Tamura, H. Thomsen, Y. Turkin, T. Wegner, I. Abramovic, S. Äkäslompolo, J. Alcuson, 
P. Aleynikov, K. Aleynikova, A. Ali, A. Alonso, G. Anda, E. Ascasibar, J.P. Bähner, S.G. Baek, M. Balden, J. Baldzuhn, M. Banduch, T. Barbui, W. Behr, C. Beidler, A. Benndorf, C. Biedermann, W. Biel, B. Blackwell, E. Blanco, M. Blatzheim, S. Ballinger, T. Bluhm, D. Böckenhoff, B. Böswirth, L.-G. Böttger, M. Borchardt, V. Borsuk, J. Boscary, H.-S. Bosch, M. Beurskens, R. Brakel, H. Brand, T. Bräuer, H. Braune, S. Brezinsek, K.-J. Brunner, R. Bussiahn, V. Bykov, J. Cai, I. Calvo, B. Cannas, A. Cappa, A. Carls, D. Carralero, L. Carraro, B. Carvalho, F. Castejon, A. Charl, N. Chaudhary, D. Chauvin, F. Chernyshev, M. Cianciosa, R. Citarella, G. Claps, J. Coenen, M. Cole, M.J. Cole, F. Cordella, G. Cseh, A. Czarnecka, K. Czerski, M. Czerwinski, G. Czymek, A. da Molin, A. da Silya, H. Damm, A. de la Pena, S. Degenkolbe, C.P. Dhard, M. Dibon, A. Dinklage, T. Dittmar, M. Drevlak, P. Drewelow, P. Drews, F. Durodie, E. Edlund, P. van Eeten, F. Effenberg, G. Ehrke, S. Elgeti, M. Endler, D. Ennis, H. Esteban, T. Estrada, J. Fellinger, Y. Feng, E. Flom, H. Fernandes, W.H. Fietz, W. Figacz, J. Fontdecaba, O. Ford, T. Fornal, H. Frerichs, A. Freund, T. Funaba, A. Galkowski, G. Gantenbein, Y. Gao, J. García Regaña, D. Gates, J. Geiger, V. Giannella, A. Gogoleva, B. Goncalves, A. Goriaev, D. Gradic, M. Grahl, J. Green, H. Greuner, A. Grosman, H. Grote, M. Gruca, C. Guerard, P. Hacker, X. Han, J.H. Harris, D. Hartmann, D. Hathiramani, B. Hein, B. Heinemann, P. Helandér, S. Henneberg, M. Henkel, J. Hernandez Sanchez, C. Hidalgo, M. Hirsch, K.P. Hollfeld, U. Höfel, A. Hölting, D. Höschen, M. Houry, J. Howard, X. Huang, Z. Huang, M. Hubeny, M. Huber, H. Hunger, K. Ida, T. Ilkei, S. Illy, B. Israeli, S. Jablonski, M. Jakubôwski, J. Jelonnek, H. Jenzsch, T. Jesche, M. Jia, P. Junghanns, J. Kacmarczyk, J.-P. Kallmeyer, U. Kamionka, H. Kasahara, W. Kasparek, Y.O. Kazakov, N. Kenmochi, C. Killer, A. Kirschner, R. Kleiber, J. Knauer, M. Knaup, A. Knieps, T. Kobarg, G. Kocsis, F. Köchl, Y. Kolesnichenko, A. Könies, R. König, P. Kornejew, J.-P. Koschinsky, F. Köster, M. Krämer, R. Krampitz, A. Krämer-Flecken, N. Krawczyk, T. Kremeyer, J. Krom, M. Krychowiak, I. Ksiazek, M. Kubkowska, G. Kühner, T. KurkiSuonio, P.A. Kurz, S. Kwak, M. Landreman, P. Lang, R. Lang, A. Langenberg, S. Langish, H. Laqua, R. Laube, S. Lazerson, C. Lechte, M. Lennartz, W. Leonhardt, C. Li, C. Li, Y. Li, Y. Liang, C. Linsmeier, S. Liu, J.-F. Lobsien, D. Loesser, J. Loizu Cisquella, J. Lore, A. Lorenz, M. Losert, A. Lücke, A. Lumsdaine, V, Lutsenko, H. Maaßberg, O. Marchuk, J.H. Matthew, S. Marsen, M. Marushchenko, S. Masuzaki, D. Maurer, M. Mayer, K. McCarthy, P. McNeely, A. Meier, D. Mellein, B. Mendelevitch, P. Mertens, D. Mikkelsen, A. Mishchenko, B. Missal, J. Mittelstaedt, T. Mizuuchi, A. Mollen, V. Moncada, T. Mönnich, T. Morisaki, D. Moseev, S. Murakami, G. Náfrádi, M. Nagel, D. Naujoks, H. Neilson, R. Neu, O. Neubauer, U. Neuner, T. Ngo, D. Nicolai, S.K. Nielsen, H. Niemann, T. Nishizawa, R. Nocentini, C. Nührenberg, J. Nührenberg, S. Obermayer, G. Offermanns, K. Ogawa, J. Ölmanns, J. Ongena, J.W. Oosterbeek, G. Orozco, M. Otte, L. Pacios Rodriguez, N. Panadero, N. Panadero Alvarez, D. Papenfuß, S. Paqay, E. Pasch, A. Pavone, E. Pawelec, T.S. Pedersen, G. Pelka, V. Perseo, B. Peterson, D. Pilopp, S. Pingel, F. Pisano, B. Plaum, G. Plunk, P. Pölöskei, M. Porkolab, J. Proll, M.-E. Puiatti, A. Puig Sitjes, F. Purps, M. Rack, S. Récsei, A. Reiman, F. Reimold, D. Reiter, F. Remppel, S. Renard, R. Riedl, J. Riemann, K. Risse, V. Rohde, H. Röhlinger, M. Romé, D. Rondeshagen, P. Rong, B. Roth, L. Rudischhauser, K. Rummel, T. Rummel, A. Runov, N. Rust, L. Ryc, S. Ryosuke, R. Sakamoto, M. Salewski, A. Samartsev, E. Sanchez, F. Sano, S. Satake, J. Schacht, G. Satheeswaran, F. Schauer, T. Scherer, J. Schilling, A. Schlaich, G. Schlisio, F. Schluck, K.-H. Schlüter, J. Schmitt, H. Schmitz, O. Schmitz, S. Schmuck, M. Schneider, W. Schneider, P. Scholz, R. Schrittwieser, M. Schröder, T. Schröder, R. Schroeder, H. Schumacher, B. Schweer, E. Scott, S. Sereda, B. Shanahan, M. Sibilia, P. Sinha, S. Sipliä, C. Slaby, M. Sleczka, H. Smith, W. Spiess, D.A. Spong, A. Spring, R. Stadler, M. Stejner, L. Stephey, U. Stridde, C. Suzuki, J. Svensson, V. Szabó, T. Szabolics, T. Szepesi, Z. SzökefalviNagy, A. Tancetti, J. Terry, J. Thomas, M. Thumm, J.M. Travere, P. Traverso, J. Tretter, H. Trimino Mora, H. Tsuchiya, T. Tsujimura, S. Tulipán, B. Unterberg, I. Vakulchyk, S. Valet, L. Vano, B. van Milligen, A.J. van Vuuren, L. Vela, J.-L. Velasco, M. Vergote, M. Vervier, 
W7-X BEAMS3D Validation

N. Vianello, H. Viebke, R. Vilbrandt, A. Vorköper, S. Wadle, F. Wagner, E. Wang, N. Wang, Z. Wang, F. Warmer, T. Wauters, L. Wegener, J. Weggen, Y. Wei, G. Weir, J. Wendorf, U. Wenzel, A. Werner, A. White, B. Wiegel, F. Wilde, T. Windisch, M. Winkler, A. Winter, V. Winters, S. Wolf, R.C. Wolf, A. Wright, G. Wurden, P. Xanthopoulos, H. Yamada, I. Yamada, R. Yasuhara, M. Yokoyama, M. Zanini, M. Zarnstorff, A. Zeitler, D. Zhang, H. Zhang, J. Zhu, M. Zilker, A. Zocco, S. Zoletnik, and M. Zuin. Overview of first wendelstein 7-x highperformance operation. Nuclear Fusion, 59(11):112004, jun 2019.

[2] Samuel A. Lazerson. Stellopt. https://www.osti.gov/doecode/biblio/12551.

[3] Matthew McMillan and Samuel Aaron Lazerson. BEAMS3D Neutral Beam Injection Model. Plasma Physics and Controlled Fusion, 56(9):095019, July 2014.

[4] Norbert Rust, Bernd Heinemann, Boris Mendelevitch, Alan Peacock, and Michael Smirnow. W7X neutral-beam-injection: Selection of the NBI source positions for experiment start-up. Fusion Engineering and Design, 86(6-8):728-731, October 2011.

[5] M C Zarnstorff, A Weller, Joachim Geiger, E Fredrickson, Stuart R Hudson, J Knauer, Allan H Reiman, A Dinklage, G Y Fu, and Long-Poe Ku. Equilibrium and stability of high-beta plasmas in wendelstein 7-as. Nuclear Fusion, 2005.

[6] Samuel Aaron Lazerson, David A Gates, D Monticello, George Hutch Neilson, N Pomphrey, Allan H Reiman, Satoru Sakakibara, and Yasuhiro Suzuki. Equilibrium Reconstruction on the Large Helical Device. In 38th EPS Conference on Plasma Physics, pages 1-4, July 2011.

[7] Samuel Aaron Lazerson and the DIII-D Team. Three-dimensional equilibrium reconstruction on the DIII-D device. Nuclear Fusion, 55(02):023009, January 2015.

[8] Samuel Aaron Lazerson and Ian T Chapman. STELLOPT modeling of the 3D diagnostic response in ITER. Plasma Physics and Controlled Fusion, 2013.

[9] Steven P Hirshman and J C Whitson. Steepest-descent moment method for three-dimensional magnetohydrodynamic equilibria. Physics of Fluids, 26(12):3553-3568, December 1983.

[10] Samuel Aaron Lazerson, Joaquim Loizu, Steven P Hirshman, and Stuart R Hudson. Verification of the ideal magnetohydrodynamic response at rational surfaces in the VMEC code. Physics of Plasmas, 23(1):012507, January 2016.

[11] Samuel Aaron Lazerson, S Sakakibara, and Yasuhiro Suzuki. A magnetic diagnostic code for 3D fusion equilibria. Plasma Physics and Controlled Fusion, 55(2):025014, January 2013.

[12] N B Marushchenko, Y Turkin, and Henning Maaßberg. Ray-tracing code TRAVIS for ECR heating, EC current drive and ECE diagnostic. Computer Physics Communications, 185(1):165176, January 2014.

[13] Oliver Ford, Lilla Vanó, Aurturo Alonso, Jugen Baldzuhn, Marc Beurskens, Christoph Biedermann, Sergey Bozhenkov, Fucherts Golo, Benedikt Geiger, Dirk Hartmann, RJE Jaspers, A Kappatou, A Langenber, Samuel Lazerson, Rachel McDermott, Paul McNeely, TWC Neelis, Novimir Pablant, Eckhard Pasch, Norbert Rust, R Schroeder, Evan R. Scott, Haken Smith, Thomas Wegner, Robert C. Wolf, and the W7-X Team. Charge Exchange Recombination Spectroscopy at Wendelstein 7-X. submitted to Rev. Sci. Inst., January 2020.

[14] N A Pablant, M Bitter, R Burhenn, L Delgado-Aparicio, R Ellis, D Gates, M Goto, K W Hill, A Langenberg, S Lazerson, M Mardenfeld, S Morita, G H Neilson, T Oishi, and T S Pedersen. Measurement of core plasma temperature and rotation on w7-x made available by the x-ray imaging crystal spectrometer (xics). In 41st EPS Conf. on Plasma Phys., page P1.076, 72014.

[15] M Endler, B Brucker, Victor Bykov, A Cardella, A Carls, F Dobmeier, A Dudek, J Fellinger, Joachim Geiger, K Grosser, O Grulke, D Hartmann, D Hathiramani, K Höchel, M Köppen, R Laube, U Neuner, X Peng, K Rahbarnia, K Rummel, T Sieber, S Thiel, A Vorköper, A Werner, T Windisch, and M Y Ye. Fusion Engineering and Design. Fusion Engineering and Design, 100:468-494, November 2015.

[16] K Rahbarnia, H Thomsen, U Neuner, J Schilling, Joachim Geiger, G Fuchert, Tamara Andreeva, M Endler, D Hathiramani, T Bluhm, M Zilker, B B Carvalho, A Werner, and Wendelstein 7-X Team. Diamagnetic energy measurement during the first operational phase at the Wendelstein 
W7-X BEAMS3D Validation

7-X stellarator. Nuclear Fusion, 58(9):096010-10, July 2018.

[17] E Pasch, M N A Beurskens, Sergey A Bozhenkov, G Fuchert, J Knauer, Robert C Wolf, and W7-X Team. The Thomson scattering system at Wendelstein 7-X. Review of Scientific Instruments, 87(11):11E729-5, September 2016.

[18] Sergey A Bozhenkov, M Beurskens, A Dal Molin, G Fuchert, E Pasch, M R Stoneking, M Hirsch, U Höfel, J Knauer, J Svensson, H Trimino Mora, and Robert C Wolf. The Thomson scattering diagnostic at Wendelstein 7-X and its performance in the first operation phase. Journal of Instrumentation, 12(10):P10004-P10004, October 2017.

[19] Matthias Hirsch, Udo Höfel, Johan Willem Oosterbeek, Neha Chaudhary, Joachim Geiger, HansJürgen Hartfuss, Walter Kasparek, Nikolai Marushchenko, Boudewijn van Milligen, Burkhard Plaum, Torsten Stange, Jakob Svensson, Hayato Tsuchiya, Dietmar Wagner, Gavin McWeir, Robert Wolf, and W7-X Team. ECE Diagnostic for the initial Operation of Wendelstein 7-X. EPJ Web of Conferences, 203((7)):03007-7, March 2019.

[20] A Pavone, U Hergenhahn, Maciej Krychowiak, U Hoefel, S Kwak, J Svensson, P Kornejew, V Winters, R Koenig, M Hirsch, K J Brunner, E Pasch, J Knauer, G Fuchert, E R Scott, M Beurskens, F Effenberg, D Zhang, O Ford, L Vano, and Robert C Wolf. Measurements of visible bremsstrahlung and automatic Bayesian inference of the effective plasma charge Zeffat W7-X. Journal of Instrumentation, 14(10):C10003-C10003, October 2019.

[21] Novimir A Pablant, A Langenberg, A Alonso, Craig D Beidler, M Bitter, Sergey A Bozhenkov, R Burhenn, M Beurskens, L Delgado-Aparicio, A Dinklage, G Fuchert, David A Gates, Joachim Geiger, K W Hill, U Höfel, M Hirsch, J Knauer, A Kramer-Flecken, M Landreman, Samuel Aaron Lazerson, Henning Maaßberg, O Marchuk, S Massidda, George Hutch Neilson, E Pasch, S Satake, J Svennson, P Traverso, Y Turkin, P Valson, J L Velasco, G Weir, T Windisch, Robert C Wolf, M Yokoyama, D Zhang, and W7-X Team. Core radial electric field and transport in Wendelstein 7-X plasmas. Physics of Plasmas, 25(2):022508-8, February 2018.

[22] S Äkäslompolo, P Drewelow, Y Gao, A Ali, O Asunta, Sergey A Bozhenkov, J Fellinger, O P Ford, N den Harder, D Hartmann, M W Jakubowski, P McNeely, H Niemann, F Pisano, Norbert Rust, A Puig Sitjes, M Sleczka, A Spanier, and Robert C Wolf. Validating fast-ion wall-load IR analysis-methods against W7-X NBI empty-torus experiment. Journal of Instrumentation, 14(07):P07018-P07018, July 2019.

[23] L. Stagner, B. Geiger, and W.W. Heidbrink. FIDASIM: A Neutral Beam and Fast-ion Diagnostic Modeling Suite.

[24] W W Heidbrink, D Liu, Y Luo, E Ruskov, and Benedikt Geiger. A Code that Simulates Fast-Ion D $\alpha$ and Neutral Particle Measurements. Communications in Computational Physics, 10(3):716741, August 2015.

[25] David Pfefferlé. Energetic ion dynamics and confinement in 3D saturated MHD configurations. $\mathrm{PhD}$ thesis, EPFL, Lausanne, 2015.

[26] O Asunta, J Govenius, R Budny, M Gorelenkova, G Tardini, T Kurki-Suonio, A Salmi, and S Sipilä. Modelling neutral beams in fusion devices: Beamlet-based model for fast particle simulations. Computer Physics Communications, 188:33-46, January 2015.

[27] H P Summers, M G O'Mullane, A D Whiteford, N R Badnell, and S D Loch. ADAS: Atomic data, modelling and analysis for fusion. In ATOMIC AND MOLECULAR DATA AND THEIR APPLICATIONS: 5th International Conference on Atomic and Molecular Data and Their Applications (ICAMDATA), pages 239-248. AIP.

[28] S Suzuki, T Shirai, M Nemoto, K Tobita, H Kubo, T Sugie, A Sakasai, and Y Kusama. Attenuation of high-energy neutral hydrogen beams in high-density plasmas. Plasma Physics and Controlled Fusion, 40(12):2097-2111, January 1999.

[29] Sergey Bozhenkov and the W7-X Team. High-performance pellet experiments in Wendelstein 7-X. submitted to Nuclear Fusion, January 2020. 